- Notre Dame Journal of Formal Logic

Volume V, Number 4, October 1964

\title{
CONTRIBUTIONS TO SYNTAX, SEMANTICS, AND THE PHILOSOPHY OF SCIENCE
}

\author{
ROLF SCHOCK
}

In the recent literature of the philosophy of science, much space has been given to the problem of analyzing theories of the deductive and natural sciences in a way which makes explicit some of the syntactic and semantic features which seem to be implicitly present in their structures. This paper is concerned with the same problem; however, some other problems of syntax and semantics are touched upon along the way. After some preliminaries, a very general method of constructing symbolic languages is introduced. The resulting languages are interpreted in either empty or nonempty sets and so in a way which permits them to contain terms which stand for nothing at all and an existence predicate which is not applicable to all terms. Also, certain variable binders are interpreted in a way which leaves open the possibility of interpreting them modally and a semantic operation of degree of truth for arbitrary formulas is introduced. An axiomless logic commensurate with a semantics of this kind and so one which contains a new treatment of definite descriptions is then constructed and shown to be both sound and semantically complete. Finally, theories and various concepts pertaining to theories are defined on the basis of this syntax and semantics. Among the concepts are operations which assign degrees of confirmation, explanatory powers, degrees of deductive simplicity, and degrees of adequacy to theories, relations and concreteness and abstractness for expressions, a relation of significance for theories, and operations which assign denotations and meanings to expressions.

\section{PRELIMINARIES}

On the assumption that the reader is acquainted with letters, arithmetic, and the rudiments of set theory, we begin with a convention and some preliminary definitions.

Convention 1. The letters ' $a$ ' through ' $z$ ' range over all existing entities.

Definition 1. If $m$ is a natural number, then $x$ is an $m$-term sequence just in case one of the following conditions is satisfied:

(1) $m=0$ and $x=$ the empty set 
(2) $m \neq 0$ and $x$ is a function whose domain is the set of all positive integers not greater than $m$.

Definition 2. $x$ is a finite sequence just in case there is a natural number $m$ such that $x$ is an $m$-term sequence.

Definition 3. If $s$ is a finite sequence, then $r$ is an inner sequence of $s$ just in case $r$ is in every set $y$ satisfying the following conditions:

(1) for any finite sequence $t$, if $t$ is in the range of $s$, then $t$ is in $y$

(2) for any $t$ in $y$ and any $u$, if $u$ is a finite sequence and $u$ is in the range of $t$, then $u$ is in $y$.

Definition 4. $x$ occurs in $s$ just in case either $x=s$ or $s$ is a finite sequence and $x$ satisfies one of the following conditions:

(1) $x$ is in the range of $s$

(2) there is an inner sequence $t$ of $s$ such that $x$ is in the range of $t$.

Definition 5. $x$ is a denumerably infinite sequence just in case $x$ is a function whose domain is the set of all positive integers.

Definition 6 . If $s$ is a denumerably infinite sequence, then

(1) the $O^{\text {th }}$ bisection of $s=s$

(2) for any positive integer $m$, the $m^{\text {th }}$ bisection of $s=$ the $t$ satisfying the following conditions:

(a) $t$ is a denume rably infinite sequence

(b) for any $i$ in the domain of $t, t(i)=\left(\right.$ the $m-1^{\text {th }}$ bisection of $s$ ) (2i).

Definition 7. If $s$ is a denumerably infinite sequence and $m$ is a natural number, then the remainder of the $m^{\text {th }}$ bisection of $s=$ the $t$ satisfying one of the following conditions:

(1) $m=0$ and $t=$ the empty set

(2) $m \neq 0$ and $t$ satisfies the following conditions:

(a) $t$ is a denumerably infinite sequence

(b) for any $i$ in the domain of $t, t(i)=\left(\right.$ the $m-1^{\text {th }}$ bisection of $s$ ) $(2 i-1)$.

Definition 8. If $r$ and $s$ are finite sequences, then $\widehat{r s}=$ the finite sequence $t$ satisfying one of the following conditions:

(1) either $r=$ the empty set and $t=s$ or $s=$ the empty set and $t=r$

(2) $r$ and $s$ are both non-empty and $t$ satisfies the following conditions:

(a) the domain of $t=$ the set of all positive integers not greater than the greatest member of the domain of $r+$ the greatest member of the domain of $s$

(b) for any $i$ in the domain of $t$, either the difference $i$-the greatest member of the domain of $r$ is not greater than 0 and $t(i)=r(i)$ or the difference $i$-the greatest member of the domain of $r$ is greater than 0 and $t(i)=s(i$-the greatest member of the domain of $r)$.

Definition 9.

(1) $\langle w\rangle=$ the 1-term sequence $t$ such that $t(1)=w$

(2) $\langle w x\rangle=$ the 2-term sequence $t$ such that $t(1)=w$ and $t(2)=x$.

(3) $\langle w x y\rangle=$ the 3 -term sequence $t$ such that $t(1)=w, t(2)=x$, and $t(3)=y$

(4) $\langle w x y z\rangle=$ the 4-term sequence $t$ such that $t(1)=w, t(2)=x, t(3)=$ $y$, and $t(4)=z$. 
Definition 10. If $x$ and $y$ are sets, then $x$ minus $y=$ the set of all $z$ such that $z$ is a member of $x$ and $z$ is not a member of $y$.

Definition 11.

(1) $\{x\}=$ the set of all $t$ such that $t=x$

(2) $\{x y\}=$ the set of all $t$ such that either $t=x$ or $t=y$.

Definition 12. If $f$ is a function, then $f\left(\begin{array}{l}x \\ y\end{array}\right)=$ the $z$ satisfying one of the following conditions:

(1) $x$ is in the domain of $f$ and $z=$ the union of ( $f$ minus the \{pair $x$, $f(x)\})$ and $\{$ the pair $x, y\}$

(2) $x$ is not in the domain of $f$ and $x=$ the union of $f$ and the pair $x, y$ \}.

Definition 13. If $f$ is a function and $x$ is a set, then the restriction of $f$ to $x=$ the set of all $y$ such that, for some $z$ in the intersection of $x$ and the domain of $f, y=$ the pair $z, f(z)$.

\section{SYMBOLS AND EXPRESSIONS}

In this paper, if an entity is assumed to exist, then, whatever it is (a cow, a number, an angel, a god, or something else), it is taken to be eligible for symbolhood. This point of view is embodied in definition 14 .

Definition 14. $x$ is a symbol sequence just in case $x$ satisfies the following conditions:

(1) $x$ is a denumerably infinite sequence

(2) for any $i$ and $j$ in the domain of $x$, if $i \neq j$, then $x(i)$ does not occur in $x(j)$.

Thus, if we take the first term of a denumerably infinite sequence $s$ to be $<$ the Atlantic Ocean>, the second term of $s$ to be the first term of

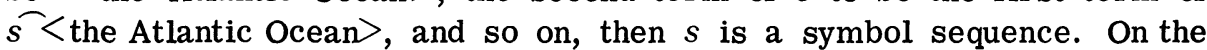
other hand, if $s$ is a symbol sequence, $i$ is in the domain of $s$, and $s(i)$ is a particular instance of the letter ' $x$ ', then there is no $j$ in the domain of $s$ such that both $j \neq i$ and either $s(j)=s(i)$ or, for some finite sequence $t$, $s(j)=\langle s(i)>t$.

By means of definitions 6 and 7 , any symbol sequence can be neatly divided up. In definitions 15 and 16, symbol sequences are divided up in a particular way and the resulting symbol sequences are taken to be sequences of variables, sequences of sentential connectives, and so on.

Definition 15. If $s$ is a symbol sequence, $i$ and $j$ positive integers, $k, l$, and $m$ are natural numbers, and either $l$ or $m$ is a positive integer, then

(1) the $i^{\text {th }}$ variable of $s=$ (the remainder of the $1^{\text {th }}$ bisection of $s$ ) $(i)$

(2) the $i^{\text {th }}$ connective of $s=$ (the remainder of the $2^{\text {th }}$ bisection of $\left.s\right)(i)$

(3) the $i^{\text {th }}$ individual constant of $s=$ (the remainder of the 3 th bisection of $s)(i)$

(4) the $i^{\text {th }} j$-place operation symbol of $s=$ (the remainder of the $j^{\text {th }}$ bisection of the remainder of the $4^{\text {th }}$ bisection of $\left.s\right)(i)$

(5) the $i^{\text {th }} j$-place predicate of $s=$ (the remainder of the $j^{\text {th }}$ bisection of the remainder of the $5^{\text {th }}$ bisection of $\left.s\right)(i)$

(6) the $i^{\text {th }} k$-place $l$-term $m$-formula term making variable binder of $s=$ (the remainder of the $k+1^{\text {th }}$ bisection of the remainder of the $l+1^{\text {th }}$ bisection of the remainder of the $m+1^{\text {th }}$ bisection of the remainder of the $6^{\text {th }}$ bisection of $\left.s\right)(i)$ 
(7) the $i^{\text {th }} k$-place $l$-term $m$-formula formula making variable binder of $s=$ (the remainder of the $k+1^{\text {th }}$ bisection of the remainder of the $l+1^{\text {th }}$ bisection of the remainder of the $m+1^{\text {th }}$ bisection of the remainder of the $7^{\text {th }}$ bisection of $\left.s\right)(i)$

(8) the $i^{\text {th }}$ auxiliary symbol of $s=$ (the remainder of the $8^{\text {th }}$ bisection of $s)(i)$.

Definition 16. If $s$ is a symbol sequence, then

(1) $x$ is a variable of $s$ just in case there is a positive integer $i$ such that $x=$ the $i^{\text {th }}$ variable of $s$

(2) $N_{s}=$ the $1^{\text {th }}$ connective of $s, \rightarrow_{s}=$ the $2^{\text {th }}$ connective of $s, \wedge_{s}=$ the $3^{\text {th }}$ connective of $s, v_{s}=$ the $4^{\text {th }}$ connective of $s$, and $\leftrightarrow_{s}=$ the $5^{\text {th }}$ connective of $s$

(3) $x$ is a standard binary connective of $s$ just in case there is a positive integer $i$ greater than 1 and less than 6 such that $x=$ the $i^{\text {th }}$ connective of $s$

(4) $x$ is an individual constant of $s$ just in case there is a positive integer $i$ such that $x=$ the $i^{\text {th }}$ individual constant of $s$

(5) for any positive integer $m$,

(a) $x$ is a $m$-place operation symbol of $s$ just in case there is a positive integer $i$ such that $x=$ the $i^{\text {th }} m$-place operation symbol of $s$

(b) $x$ is an $m$-place predicate of $s$ just in case there is a positive integer $i$ such that $x=$ the $i^{\text {th }} m$-place predicate of $s$

(f) $x$ is an operation symbol of $s$ just in case there is a positive integer $m$ such that $x$ is an $m$-place operation symbol of $s$

(7) $x$ is a predicate of $s$ just in case there is a positive integer $m$ such that $x$ is an $m$-place predicate of $s$

(8) $\mathrm{E}_{s}=$ the $1^{\text {th }} 1$-place predicate of $s$ and $\mathrm{I}_{s}=$ the $1^{\text {th }} 2$-place predicate of $s$

(9) for any natural numbers $k, l$, and $m$, if either $l$ or $m$ is a positive integer, then

(a) $x$ is a $k$-place $l$-term $m$-formula term making variable binder of $s$ just in case there is a positive integer $i$ such that $x=$ the $i^{\text {th }} k$-place $l$-term $m$-formula term making variable binder of $s$

(b) $x$ is a $k$-place $l$-term $m$-formula formula making variable binder of $s$ just in case there is a positive integer $i$ such that $x=$ the $i^{\text {th }} k$ place $l$-term $m$-formula formula making variable binder of $s$

(10) $\boldsymbol{1}_{s}=$ the $1^{\text {th }} 1$-place 0 -term 1 -formula term making variable binder of $s, \Lambda_{s}=$ the $1^{\text {th }} 1$-place 0 -term 1 -formula formula making variable binder of $s$, and $V_{s}=$ the $2^{\text {th }} 1$-place 0 -term 1 -formula formula making variable binder of $s$

(11) for any natural number $k, x$ is a $k$-place variable binder of $s$ just in case there are natural numbers $l$ and $m$ such that

(a) either $l$ or $m$ is a positive integer

(b) $x$ is a $k$-place $l$-term $m$-formula term making or formula making variable binder of $s$

(12) $x$ is a variable binder of $s$ just in case there is a natural number $k$ such that $x$ is a $k$-place variable binder of $s$ 
(13) show $_{s}=$ the $1^{\text {th }}$ auxiliary symbol of $s$

(14) $x$ is a pure constant of $s$ just in case either $x=N_{s}$ or $x$ is a standard binary connective of $s$ or $x=1_{s}$ or $x=\Lambda_{s}$ or $x=\vee_{s}$

(15) $x$ is a descriptive constant of $s$ just in case either $x$ is an individual constant of $s$ or $x$ is an operation symbol of $s$ or $x$ is a predicate of $s$ or $x$ is a 0 -place variable binder of $s$

(16) $x$ is a standard symbol of $s$ just in case either $x$ is a variable of $s$ or $x$ is a pure constant of $s$ or $x$ is a descriptive constant of $s$ or $x=s h o w_{s}$.

Terms, formulas, and expressions of a symbol sequence $s$ can be defined on the basis of definitions 15 and 16 .

Definition 17. If $s$ is a symbol sequence, then

(1) Klmbtpfqs just in case $l$ and $m$ are natural numbers, either $l$ or $m$ is a positive integer, $b$ is a 0 -place $l$-term $m$-formula term making variable binder of $s, p$ and $q$ are sets, $t$ is an $l$-term sequence whose range is included in $p$, and $f$ is an $m$-term sequence whose range is included in $q$

(2) Llmbtpfqs just in case $l$ and $m$ are natural numbers, either $l$ or $m$ is a positive integer, $b$ is a $O$-place $l$-term $m$-formula formula making variable binder of $s, p$ and $q$ are sets, $t$ is an $l$-term sequence whose range is included in $p$, and $f$ is an $m$-term sequence whose range is included in $q$

(3) $p$ is closed under term making in $s$ from $q$ just in case the following conditions are satisfied:

(a) $p$ and $q$ are sets

(b) every variable of $s$ is in $p$

(c) every individual constant of $s$ is in $p$

(d) for any positive integer $m, m$-place operation symbol of $s b$, and $m$-term sequence $t$, if the range of $t$ is included in $p$, then $\langle b\rangle t$ is in $p$

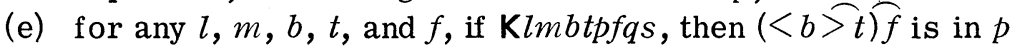

(f) for any variable of $s v$ and $f$ in $q,\left\langle\mathbf{1}_{s} v f\right\rangle$ is in $p$

(4) $q$ is closed under formula making in $s$ from $p$ just in case the following conditions are satisfied:

(a) $p$ and $q$ are sets

(b) for any positive integer $m, m$-place predicate of $s b$, and $m$ term sequence $t$, if the range of $t$ is included in $p$, then $\langle b\rangle t$ is in $q$

(c) for any $f$ in $q,\left\langle N_{s} f\right\rangle$ is in $q$

(d) for any $f$ and $g$ in $q$ and standard binary connective of $s b$, $\langle f b g\rangle$ is in $q$

(e) for any $l, m, b, t$, and $f$, if Llmbtpfqs, then $(\langle b>t) \widehat{f}$ is in $q$

(f) for any variable of $s v$ and $f$ in $q,\left\langle\wedge_{s} v f\right\rangle$ and $\left\langle\vee_{s} v f\right\rangle$ are in $q$

(5) $u$ is a 0 -level term of $s$ just in case either $u$ is a variable of $s$ or $u$ is an individual constant of $s$

(6) if $k$ is a positive integer, then $u$ is a $k$-level term of $s$ just in case for any $p$, if $p$ is closed under term making in $s$ from the set of all $k-1$ level formulas of $s$, then $u$ is in $p$

(7) if $k$ is a natural number, then $h$ is a $k$-level formula of $s$ just in case, for any $q$, if $q$ is closed under formula making in $s$ from the set of all $k$-level terms of $s$, then $h$ is in $q$ 
(8) $u$ is a term of $s$ just in case, for some natural number $k, u$ is a $k$ level term of $s$

(9) $h$ is a formula of $s$ just in case, for some natural number $k, h$ is a $k$-level formula of $s$.

This cumbersome, oscillating, and multiply recursive simultaneous definition characterizes the terms and formulas of a symbol sequence $s$ by the way they are built up out of the members of the range of $s$ which occur in them. The usefulness of this definition will be come apparent later.

Notice that, for any symbol sequence $s$, many members of the range of $s$ are not standard symbols of $s$ and many connectives and variable binders of $s$ do not occur in any terms or formulas of $s$. This policy is adopted here so that the syntax and semantics of the ignored members of the range of such an $s$ can be bypassed.

Definition 18. If $s$ is a symbol sequence, then $x$ is an expression of $s$ just in case $x$ is in every set $y$ satisfying the following conditions:

(1) every standard symbol of $s$ is in $y$

(2) for any positive integer $m$ and $m$-term sequence $t$, if the range of $t$ is included in $y$, then $t$ is in $y$.

Convention 2. We sometimes write ' $E$ ' for ' $E_{s}$ ' ' $I$ ' for ' $I_{s}$ ', ' $N$ ' for ' $N_{s}$ ', ' $\rightarrow$ ' for ' $\rightarrow s^{\prime}$ ', ' $\wedge$ ' for ' $\wedge_{s}$ ', ' $v$ ' for ' $v_{s}$ ', ' $\leftrightarrow$ ' for ' $\leftrightarrow s^{\prime}$, ' $\eta$ ' for ' $1_{s}$ ', ' $\wedge$ ' for ' $\Lambda_{s}$ ', ' $\vee$ ' for ' $\vee_{s}$ ', and 'show' for 'shows'.

Convention 3. The usual conventions for the omission of parentheses in used expressions will sometimes be employed for the omission of sequence marks in the mentioned expressions of symbol sequences.

Definition 19. If $s$ is a symbol sequence and $t$ and $u$ are terms of $s$, then $t \mathbf{I}_{s} u=\left\langle\mathbf{I}_{s} t u\right\rangle$.

\section{SEMANTIC CONCEPTS}

In contemporary semantics, it has become customary to interpret expressions in a way which insures that every term stands for something and so within non-empty sets ${ }^{1}$. Although this procedure is very elegant, it leads to results which are not at all acceptable to some philosophers. For instance, a formula representing the self identity of the non-self identical thing will be true; moreover, if the entity which a term not really standing for anything stands for is taken to be the number 330 , then a formula representing the content of the statement 'the star revolving around the sun in the orbit of Mars is a number divisible both by 3 and 10' will be true under any normal interpretation.

In this paper, the interpreted expressions of any symbol sequences are interpreted in a way which allows some or even all terms of $s$ to not stand for anything and so which leaves open the possibility of interpreting these terms within the empty set $^{2}$. This interpretation is given both to avoid the odd situations mentioned in the preceding paragraph and for the sake of definition 25.

Definition 20. If $s$ is a symbol sequence, $x$ is a set, and either $t$ is a term of $s$ or $t$ is a formula of $s$, then $y$ is an assigner for $t$ in $x$ with respect to $s$ just in case the following conditions are satisfied: 
(1) $y$ is a function

(2) the set of all variables of $s v$ such that $v$ occurs in $t$ is included in the domain of $y$ and the domain of $y$ is included in the set of all variables of $s$

(3) for any $v$ in the domain of $y$, either $x$ is empty and $y(v)=$ the empty ty set or $x$ is not empty and, for some $m$ in $x, y(v)=\{m\}$.

Definition 21. If $s$ is a symbol sequence, then

(1) $x$ is an interpreter with respect to $s$ just in case $x$ satisfies the following conditions:

(a) $x$ is a function

(b) the domain of $x=$ the set of all descriptive constants of $s$

(c) there is a set $y$ such that

(1) for any individual constant of $s b$, either $x(b)=$ the empty set or, for some $m$ in $y, x(b)=\{m\}$

(2) for any positive integer $m$ and any $b$,

(a) if $b$ is an $m$-place operation symbol of $s$, then

(1) $x(b)$ is a function

(2) the domain of $x(b)=$ the set of all $m$-term sequences whose ranges are included in $y$

(3) for any $t$ in the domain of $x(b)$, either $(x(b))(t)=$ the empty set or, for some $m$ in $y,(x(b))(t)=\{m\}$

(b) if $b$ is an $m$-place predicate of $s$, then $x(b)$ is included in the set of all $m$-term sequences whose ranges are included in $y$ included in $y$

(3) $x\left(E_{s}\right)=$ the set of all 1 -term sequences whose ranges are

(4) $x\left(\mathbf{I}_{s}\right)=$ the set of all 2-term sequences $t$ such that, for some $m$ in $y$, the range of $t=\{m\}$

(5) for any natural numbers $l$ and $m$, if either $l$ or $m$ is a positive integer, then, for any $b$,

iable binder of $s$, then

(a) if $b$ is a 0 -place $l$-term $m$-formula term making var-

(1) $x(b)$ is a function

(2) the domain of $x(b)=$ the set of all 2-term sequences $t$ such that $t(1)$ is an $l$-term sequence whose range is included in the set of all terms of $s$ and $t(2)$ is an $m$-term sequence whose range is included in the set of all formulas of $s$

(3) for any $t$ in the domain of $x(b)$, either $(x(b))(t)=$ the empty set or, for some $m$ in $y,(x(b))(t)=\{m\}$

(b) if $b$ is a $O$-place $l$-term $m$-formula formula making variable binder of $s$, then $x(b)$ is included in the set of all 2-term sequences $t$ such that $t(1)$ is an $l$-term sequence whose range is included in the set of all terms of $s$ and $t(2)$ is an $m$-term sequence whose range is included in the set of all formulas of $s$

(2) if $i$ is an interpreter with respect to $s$, then $U i=$ the $y$ such that $i\left(E_{s}\right)=$ the set of all 1-term sequences whose ranges are included in $y$.

Definition 22. If $s$ is a symbol sequence, $i$ is an interpreter with respect to $s, t$ is either a term of $s$ or a formula of $s$, and $a$ is anassigner for $t$ in $\mathrm{U} i$ with respect to $s$, then 
(1) if $t$ is a variable of $s$, then the interpretation of $t$ with respect to $i$ and $a=a(t)$

(2) if $t$ is an individual constant of $s$, then the interpretation of $t$ with respect to $i$ and $a=i(t)$

(3) for any positive integer $m$ and any $u$, if $u$ is an $m$-term sequence whose rarge is included in the set of all terms of $s$, then, for any $b$,

(a) if $b$ is an $m$-place operation symbol of $s$ and $t=\langle\vec{b}\rangle \vec{u}$, then one of the following conditions is satisfied:

(1) the interpretation of $t$ with respect to $i$ and $a=$ the empty set and there is no $m$-term sequence $v$ such that, for any $j$ in the domain of $v, v(j)$ is a member of the interpretation of $u(j)$ with respect to $i$ and $a$

(2) there is an $m$-term sequence $v$ such that $v(j)$ is a member of the interpretation of $u(j)$ with respect to $i$ and $a$ for any $j$ in the domain of $v$ and the interpretation of $t$ with respect to $i$ and $a=(i(b))(v)$

(b) if $b$ is an $m$-place predicate of $s$ and $t=\langle b\rangle u$, then $t$ satisfies $i$ and $a$ just in case these is an $m$-term sequence $v$ such that $v$ is a member of $i(b)$ and, for any $j$ in the domain of $v, v(j)$ is a member of the interpretation of $u(j)$ with respect to $i$ and $a$

(4) for any formula of $s f$, if $t=\left\langle N_{s} f\right\rangle$, then $t$ satisfies $i$ and $a$ just in case $f$ does not satisfy $i$ and $a$

(5) for any formulas of $s f$ and $g$,

(a) if $t=\left\langle f \rightarrow_{s} g\right\rangle$, then $t$ satisfies $i$ and $a$ just in case $f$ satisfies $i$ and $a$ ondy if $g$ satisfies $i$ and $a$

(b) if $t=\left\langle f \wedge_{s} g\right\rangle$, then $t$ satisfies $i$ and $a$ just in case both $f$ and $g$ satisfy $i$ and $a$

(c) if $t=\left\langle f v_{s} g\right\rangle$, then $t$ satisfies $i$ and $a$ just in case either $f$ or $g$ satigfies $i$ and $a$

(d) if $f=\left\langle f \leftrightarrow{ }_{s} g\right\rangle$, then $t$ satisfies $i$ and $a$ just in case either both $f$ and $g$ satisfy $i$ and $a$ or both $f$ and $g$ do not satisfy $i$ and $a$

(6) for any $l, m, b, u$, and $f$,

(e) if Klmbu (the set of all terms of $s$ ) $f$ (the set of all formulas of $s) s$ and $t=(\langle b>\vec{u})$, then the interpretation of $t$ with respect to $i$ and $a=$ $(i(b))(<u f>)$

(b) if Llmbu (the set of all terms of $s$ ) $f$ (the set of all formulas of s) $s$ and $t=(\langle b>\widehat{u} \widehat{f}$, then $t$ satisfies $i$ and $a$ just in case $\langle u f\rangle$ is a member of $i(b)$

(7) for any variable of $s v$ and formula of $s f$,

(a). if $t=\left\langle\boldsymbol{1}_{s} v f\right\rangle$, then one of the following conditions is satis-

fied:

(1) the interpretation of $t$ with respect to $i$ and $a=$ the empty set and there is no $y$ in $U i$ such that, for any $x$ in $U i, f$ satisfies $i$ and $a\left(\begin{array}{l}v \\ x\}\end{array}\right)$ just in case $x=y$

(2) there is a $y$ in $U i$ such that both $f$ satisfies $i$ and $a\left(\begin{array}{c}v \\ x \\ x\end{array}\right)$ just in case $x=y$ for any $x$ in $\mathrm{U} i$ and the interpretation of $t$ with respect to $i$ and $a=\{y\}$

(b) if $t=\left\langle\wedge_{s} v f\right\rangle$, then $t$ satisfies $i$ and $a$ just in case, for any $x$ in $U i, f$ satisfies $i$ and $a\left(\left\{\begin{array}{c}v \\ x\}\end{array}\right)\right.$ 
(c) if $t=\left\langle\mathrm{V}_{s} v f\right\rangle$, then $t$ satisfies $i$ and $a$ just in case, for some $x$ in $\mathrm{U} i, f$ satisfies $i$ and $a\left(\left\{\begin{array}{l}v \\ x\end{array}\right\}\right)$.

An intuitive account of the main properties of the just defined concepts seems to be advisable at this point. For any symbol sequence $s, \mathbf{E}_{s}, \mathbf{I}_{s}$, $N_{s}, \rightarrow_{s}, \wedge_{s}, \vee_{s}, \leftrightarrow_{s}, \boldsymbol{1}_{s}, \wedge_{s}$, and $\vee_{s}$ are interpreted so as to function more or less as the expressions 'exists,' 'is identical with,' 'not,' 'only if,' 'and,' 'or,' 'if and only if,' 'the,' 'for any,' and 'for some' of ordinary English respectively. Thus, in spite of the tradition in philosophy according to which 'exists' is not a predicate, the representative of 'exists' for $s$ is a predicate. This predicate is of philosophical importance as well as being useful for the formulation of quantifier, identity, and definite description logic for formulas in which terms interpreted to be empty occur. For instance, a formula representing the self identity of the non-self identical thing cannot satisfy any interpreter $i$ and assigner for the formula in the universe of discourse within which $i$ operates. Thus, the principle of self identity must be restricted through the addition of something like an existence condition to it. Similar restrictions must be made with respect to such logical procedures as universal instantiation and existential generalization. Also, universal generalization must be extended and the rules for descriptive phrases must be modified through the addition of something like existence clauses. All of this follows from the fact that the interpreted expressions of any symbol sequence $s$ can be interpreted within the empty set.

In addition to leading to more or less the usual kinds of interpretations for the individual constants, operation symbols, and predicates of a symbol sequence $s$, the interpreters of definition 21 also provide interpretations for the $O$-place variable binders of $s$ (including any modal $O$-place variable binders of $s$ ). These are, so to speak, interpreted as representing operations among or attributes of sequences of terms of $s$ and sequences of formulas of $s$.

On the basis of definition 22, both a fairly normal definition of truth for any formula of a symbol sequence $s$ and an unusual definition of the degree of truth of such a formula can be given.

Definition 23. If $s$ is a symbol sequence, $x$ is a set, and either $t$ is a term of $s$ or $t$ is a formula of $s$, then $y$ is a proper assigner for $t$ in $x$ with respect to $s$ just in case $y$ satisfies the following conditions:

(1) $y$ is an assigner for $t$ in $x$ with respect to $s$

(2) the domain of $y=$ the set of all variables of $s v$ such that $v$ occurs in $t$.

Definition 24. If $s$ is a symbol sequence, $i$ is an interpreter with respect to $s$, and $x$ is included in $U i$, then the reduction of $i$ for $x$ with respect to $s=$ the $y$ satisfying the following conditions:

(1) $y$ is an interpreter with respect to $s$

(2) for any individual constant of $s, b, y(b)=$ the intersection of $i(b)$ and $x$

(3) for any positive integer $m$ and $b$,

(a) if $b$ is an $m$-place operation symbol of $s$, then, for any $t$ in the domain of $y(b),(y(b))(t)=$ the intersection of $(i(b))(t)$ and $x$ 
(b) if $b$ is an $m$-place predicate of $s$, then $y(b)=$ the set of all $t$ such that $t$ is in $i(b)$ and the range of $t$ is included in $x$

(4) for any natural numbers $l$ and $m$, if either $l$ or $m$ is a positive integer, then, for any $b$.

(a) if $b$ is a $O$-place $l$-term $m$-formula term making variable binder of $s$, then, for any $t$ in the domain of $y(b),(y(b))(t)=$ the intersection of $(i(b))(t)$ and $x$

(b) if $b$ is a 0 -place $l$-term $m$-formula formula making variable binder of $s$, then $y(b)=i(b)$.

Definition 25. If $s$ is a symbol sequence, $f$ is a formula of $s$, and $i$ is an interpreter with respect to $s$, then

(1) $f$ is true with respect to $i$ and $s$ just in case, for any proper assigner for $f$ in $U i$ with respect to $s a, f$ satisfies $i$ and $a$

(2) for any finite subset of $U i x$, the degree of truth of $f$ in $x$ with respect to $i$ and $s=$ the $y$ such that, for some $m$ and $n$,

(a) $m=$ the number of members of the set of all proper assigners for $f$ in $x$ with respect to $s a$ such that $f$ satisfies the reduction of $i$ for $x$ with respect to $s$ and $a$

(b) $n=$ the number of members of the set of all proper assigners for $f$ in $x$ with respect to $s$

(c) $y=$ the fraction $m / n$.

From definition 25, it follows that, if its condition is satisfied and $U i$ is finite, then $f$ is true with respect to $i$ and $s$ just in case the degree of truth of $f$ in $U i$ with respect to $i$ and $s=1$; hence, the degree of truth of $f$ in $U i$ with respect to $i$ and $s$ need not be $O$ for $f$ to not be true with respect to $i$ and $s$. Naturally, this correspondence of truth and maximal degree of truth need not hold if $U i$ is infinite since the degree of truth of $f$ in $U i$ with respect to $i$ and $s$ is then not defined.

What is embodied in part (2) of definition 25 may be clarified further by means of some examples. Let $s$ be a symbol sequence and let $p$ be an interpreter with respect to $s$ such that $U_{p}=$ the set of all natural numbers. Suppose that the formulas of $s$ have among them the representatives $e, f, g$, $h, i, j, k, l, m$, and $n$ of the statements of English ' 1 is a number,' ' $O$ is a number,' 'there is an $x$ greater than 1,' 'there is an $x$ greater than $y$,' ' $x$ is greater than 1, ' $x$ is less than 3,' 'every $x$ is not greater than 2,' 'every $x$ is greater than 0 ,' 'every $x$ is not smaller than $y$,' and 'the $x$ such that $x$ is greater than $y$ exists' respectively. We suppose both that these are expressed in a normal way, i.e., by means of appropriate individual constants, variable binders, variables, and predicates of $s$, and normally interpreted by $p$. Let $d=$ the set of all positive integers smaller than 3 , let $q=$ the reduction of $p$ for $d$ with respect to $s$, and let $t=$ the function whose domain is the set whose members are $e$ through $n$ and which assigns to any $y$ in its domain the degree of truth of $y$ in $d$ with respect to $p$ and $s$. If we write 'is true' for 'is true with respect to $p$ and $s$,' then we have the following:

(1) $t(e)=1$ and $e$ is true

(2) $t(f)=0$ and $f$ is true

(3) $t(g)=1$ and $g$ is true 
(4) $t(h)=1 / 2$ and $h$ is true

(5) $t(i)=1 / 2$ and $i$ is not true

(6) $t(j)=0$ and $j$ is not true

(7) $t(k)=1$ and $k$ is not true

(8) $t(l)=0$ and $l$ is not true

(9) $t(m)=1 / 2$ and $m$ is not true

(10) $t(n)=1 / 2$ and $n$ is not true

Since no variable of $s$ occurs in either $e$ or $f$, the only proper assigner for either one in $d$ with respect to $s$ is the empty set. Since both $e$ and $f$ are true whereas $O$ is not in $d$ and so only $e$ satisfies $q$ and the empty set, we have (1) and (2).

There are only two proper assigners for $g, i, j, k$, and $l$ in $d$ with respect to $s$. Since 3 and $O$ are not in $d, j$ and $l$ do not satisfy $q$ and either one of them. Then, since $g$ and $k$ satisfy $q$ and either one of them, since $i$ satisfies $q$ and only the one of them which assigns $\{2\}$ to the variable of $s$ occuring in $i$ which represents ' $x$,' and since only $g$ is true, we have (3) and (5) through (8).

There are only four proper assigners for $h, m$, and $n$ in $d$ with respect to $s$. Since $h, m$, and $n$ satisfy $q$ and the two of them which assign $\{1\}$ to the variable of $s$ occurring in $h, m$, and $n$ which represents ' $y$ ' and since only $h$ is true, we have (4), (9), and (10).

For the sake of some further remarks on this semantics, validity, tautologousness, freedom, and proper substitution must be defined.

Definition 26. If $s$ is a symbol sequence and $f$ is a formula of $s$, then

(1) $f$ is valid with respect to $s$ just in case, for any $i$, if $i$ is an interpreter with respect to $s$, then $f$ is true with respect to $i$ and $s$

(2) $f$ is a tautology of $s$ just in case, for any $g, g(f)=1$ if $g$ satisfies the following conditions:

(a) $g$ is a function

(b) the domain of $g=$ the set of all formulas of $s$

(c) the range of $g=\{01\}$

(d) for any $h$ and $i$ in the domain of $g$,

(1) $g\left(<N_{s} h>\right)=1-g(h)$

(2) $g\left(<h \rightarrow_{s} i>\right)=$ the smallest member of $\{1(1-g(h))+g(i)\}$

(3) $g\left(\left\langle h \wedge_{s} i>\right)=\right.$ the smallest member of $\{g(h) g(i)\}$

(4) $g\left(\left\langle h \vee_{s} i\right\rangle\right)=$ the greatest member of $\{g(h) g(i)\}$

(5) $g\left(<h \leftrightarrow{ }_{s} i>\right)=(1$-the greatest member of $\{g(h) g(i)\})+$ the smallest member of $\{g(h) g(i)\}$.

Definition 27. If $s$ is a symbol sequence and $a$ is a term of $s$, then

(1) for any $b$, if either $b$ is a variable of $s$ or $b$ is an individual constant of $s$, then $a$ is free in $b$ just in case $a=b$

(2) for any positive integer $m, m$-place operation symbol or predicate of $s b$, and $m$-term sequence $t$, if the range of $t$ is included in the set of all terms of $s$, then $a$ is free in $\langle b>t$ just in case either $a=\langle b\rangle t$ or, for some $i$ in the domain of $t, a$ is free in $t(i)$

(3) for any formula of $s f, a$ is free in $\left\langle N_{s} f\right\rangle$ just in case $a$ is free in $f$

(4) for any formulas of $s f$ and $g$ and standard binary connective of $s b$, $a$ is free in $\langle f b g\rangle$ just in case either $a$ is free in $f$ or $a$ is free in $g$ 
(5) for any $l, m, b, t$, and $f$, if either Klmbt (the set of all terms of $s) f$ (the set of all formulas of $s$ ) $s$ or Llmbt (the set of all terms of $s$ ) $f$ (the set of all formulas of $s) s$, then $a$ is free in $(\langle b>t) \widehat{f}$ just in case $a=(\langle b>t) \widehat{f}$

(6) for any $b$, if either $b=1_{s}$ or $b=\wedge_{s}$ or $b=\vee_{s}$, then, for any variable of $s v$ and formula of $s f, a$ is free in $\langle b v f\rangle$ just in case either $a=$ $<b v f>$ or both $a$ is free in $f$ and $v$ is not free in $a$.

Notice that, for any symbol sequence $s$, the $O$-place variable binders of $s$ bind all of the terms of $s$ in their scopes ${ }^{3}$.

Definition 28. If $s$ is a symbol sequence, $a$ and $b$ are terms of $s$, and either $c$ is a term of $s$ or $c$ is a formula of $s$, then

(1) if $a=c$, then the result of properly substituting $b$ for $a$ in $c$ in $s=b$

(2) if $a \neq c$, then

(a) if either $c$ is a variable of $s$ or $c$ is an individual constant of $s$, then the result of properly substituting $b$ for $a$ in $c$ in $s=c$

(b) for any positive integer $m, m$-place operation symbol or predicate of $s d$, and $m$-term sequence $t$, if the range of $t$ is included in the set of all terms of $s$ and $c=\langle\vec{d}\rangle \vec{t}$, then the result of properly substituting $b$ for $a$ in $c$ in $s=\langle d\rangle$ the $m$-term sequence $u$ such that, for any $i$ in the domain of $u, u(i)=$ the result of properly substituting $b$ for $a$ in $t(i)$ in $s$

(c) for any formula of $s f$, if $c=\left\langle N_{s} f\right\rangle$, then the result of properly substituting $b$ for $a$ in $c$ in $s=\left\langle N_{s}\right.$ the result of properly substituting $b$ for $a$ in $f$ in $s>$

(d) for any formulas of $s f$ and $g$ and standard binary connective of $s d$, if $c=\langle f d g\rangle$, then the result of properly substituting $b$ for $a$ in $c$ in $s=<$ the result of properly substituting $b$ for $a$ in $f$ in $s d$ the result of properly substituting $b$ for $a$ in $g$ in $s>$

(e) for any $l, m, d, t$, and $f$, if both either $\mathrm{K} l m d t$ (the set of all terms of $s$ ) $f$ (the set of all formulas of $s$ ) $s$ or Llmdt (the set of all terms of $s$ ) $f$ (the set of all formulas of $s) s$ and $c=(\langle d>t) \widehat{f}$, then the result properly substituting $b$ for $a$ in $c$ in $s=c$

(f) for any $d$, if either $d=1_{s}$ or $d=\wedge_{s}$ or $d=\vee_{s}$, then, for any variable of $s b$ and formula of $s f$, if $c=\langle d v f\rangle$, then the result of properly substituting $b$ for $a$ in $c$ in $s=$ the 3 -term sequence $t$ satisfying one of the following conditions:

(1) $a$ is free in $\langle d v f\rangle$ and either $v$ is not free in $b$ and $t=$ $<d v$ the result of properly substituting $b$ for $a$ in $f$ in $s>$ or $v$ is free in $b$ and, for some positive integer $m$, either the $m^{\text {th }}$ variable of $s$ occurs in $\langle d v f\rangle$ or the $m^{\text {th }}$ variable of $s$ occurs in $b$, there is no positive integer $n$ greater than $m$ such that either the $n^{\text {th }}$ variable of $s$ occurs in $\langle d v f\rangle$ or the $n^{\text {th }}$ variable of $s$ occurs in $b$, and $t=\left\langle d\right.$ the $m+1^{\text {th }}$ variable of $s$ the result of properly substituting $b$ for $a$ in the result of properly substituting the $m+1^{\text {th }}$ variable of $s$ for $v$ in $f$ in $s$ in $s>$

(2) $a$ is not free in $\langle d v f\rangle$ and $t=\langle d v f\rangle$.

On the basis of these definitions, it can be shown that, if $s$ is a symbol sequence, $m$ is a positive integer, $a$ is an $m$-place operation symbol of $s, b$ is an $m$-place predicate of $s, v, w$, and $x$ are variables of $s, r$ and $t$ are terms of $s, u$ is an $m$-term sequence whose range is included in the set of all terms of $s, f, g, h, i$, and $j$ are formulas of $s, x$ is not free in $h, x \neq v, g$ is a tautology of $s, q$ is in the range of $u$, and both $i \rightarrow j$ and $i$ are valid with respect to $s$, then the following formulas are valid with respect to $s$ : 
(1) $\mathrm{E}<a>u \rightarrow \mathrm{E} q$

(2) $\langle b>\widehat{\sim} \rightarrow \mathrm{E} q$

(3) $\vee v f \rightarrow \mathbf{E} w$

(4) $g$

(5) $\wedge v f \wedge \mathrm{E} t \rightarrow$ the result of properly substituting $t$ for $v$ in $f$ in $s$

(6) $\wedge x<h \rightarrow f>\rightarrow\langle h \rightarrow \wedge x f>$

(7) $\vee v f \leftrightarrow N \wedge v N f$

(8) $\langle r \mathrm{I} t \vee\langle N E r \wedge N \mathrm{E} t\rangle \wedge \wedge$ the result of properly substituting $r$ for $t$ in $f$ in $s \rightarrow f$

(9) $\mathrm{E} t \rightarrow t \mathbf{I} t$

(10) $\vee x \wedge v<h \leftrightarrow v \mathbf{I} x>\rightarrow$ the result of properly substituting $1 v h$ for $v$ in $h$ in $s \wedge \mathrm{E}\} h$

(11) Eq $v h \rightarrow \vee x \wedge v<h \leftrightarrow v \mathbf{I} x>$

(12) $j$

(13) $\wedge v i$.

The proof for this assertion is tedious although simple and so will not be given here $e^{4}$. In the next section, an axiomless deductive system ${ }^{5}$ according to which (1) through (13) are theorems if $i \rightarrow j$ and $i$ are will be constructed.

Notice that these formulas are true with respect to an interpreter $i$ and a symbol sequence $s$ even if $\boldsymbol{U} i$ is empty. Notice also that, for any formula of $s f$ and finite subset of $\mathrm{U} i x$, if $f$ is valid with respect to $s$, then the degree of truth $f$ and $N f$ in $x$ with respect to $i$ and $s$ are 1 and $O$ respectively.

\section{DEDUCTIVE SYSTEMS}

A set of axioms, a set of assumption rules, a set of inference rules, and a set of proof methods can together be understood as making up a deductive system. In definitions 29 through 33 , this point of view is adopted and made exact.

Definition 29. If $s$ is a symbol sequence, then $x$ is an assumption rule of $s$ just in case $x$ satisfies the following conditions:

(1) $x$ is a function

(2) the domain of $x=$ the set of all $y$ such that, for some formula of $s f$, $y=\left\langle\right.$ show $\left._{s} f\right\rangle$

(3) for any $y$ in the domain of $x$, there is a formula of $s f$ such that $x(y)$ is included in $\{f\}$.

Definition 30. If $s$ is a symbol sequence, then $x$ is a line of $s$ just in case $x$ satisfies one of the following conditions:

(1) $x$ is a formula of $s$

(2) for some formula of $s f, x=\left\langle\right.$ show $\left._{s} f\right\rangle$.

Definition 31. If $s$ is a symbol sequence, then $x$ is an inference rule of $s$ just in case $x$ satisfies the following conditions:

(1) $x$ is a function

(2) the domain of $x=$ the set of all finite sequences whose ranges are included in the set of all lines of $s$

(3) for any $y$ in the domain of $x, x(y)$ is included in the set of all formulas of $s$. 
Definition 32. If $s$ is a symbol sequence, then $x$ is a proof method of $s$ just in case $x$ satisfies the following conditions:

(1) $x$ is a function

(2) the domain of $x=$ the set of all finite sequences whose ranges are included in the set of all lines of $s$

(3) for any $y$ in the domain of $x, x(y)$ is included in the set of all $z$ such that, for some formula of $s f$ and $m$ in the domain of $y$, the following conditions are satisfied:

(a) $y(m)=\left\langle\right.$ show $\left._{s} f\right\rangle$

(b) there is no $n$ in the domain of $y$ greater than $m$ such that, for some formulas of $s g, y(n)=\left\langle\right.$ show $\left._{s} g\right\rangle$

(c) $z=$ (the restriction of $y$ to the set of all $l$ in the domain of $y$ such that $l$ is smaller than $m)\left(\begin{array}{c}m \\ f\end{array}\right)$.

Definition 33. If $s$ is a symbol sequence, then $x$ is a deductive system of $s$ just in case $x$ satisfies the following conditions:

(1) $x$ is a 4 -term sequence

(2) $x(1)$ is included in the set of all formulas of $s$

(3) $x(2)$ is included in the set of all assumption rules of $s$

(4) $x(3)$ is included in the set of all inference rules of $s$

(5) $x(4)$ is included in the set of all proof methods of $s$.

On the basis of this view of what a deductive system is, a theorem of a deductive system $d$ can be understood as a formula into which the command to show that formula can be transformed by means of the axioms, assumption rules, inference rules, and proof methods of $d$.

Definition 34. If $s$ is a symbol sequence and $d$ is a deductive system of $s$, then $x$ is a proof in $s$ by $d$ just in case $x$ is in every set $y$ satisfying the following conditions:

(1) for any formula of $s f$,

(a) 《shows $f \gg$ is in $y$

(b) for any $q$ in $y, q$ q $\ll$ shows $f \gg$ is in $y$

(c) for any $q$ in $y$, if $q$ (the greatest member of the domain of $q$ ) = $<$ show $_{s} f>$, then, for any $a$ in $d(2)$ and $g$ in $a\left(\left\langle\right.\right.$ show $\left._{s} f>\right), \widetilde{q<g}>$ is in $y$

(2) for any $a$ in $d(1)$ and $q$ in $y, \widetilde{q<a>}$ is in $y$

(3) for any $q$ in $y, r$ in $d(3)$, and $f$ in $r(q), \widehat{q<f>}$ is in $y$

(4) for any $q$ in $y$ and $p$ in $d(4)$, every member of $p(q)$ is in $y$.

Definition 35. If $s$ is a symbol sequence and $d$ is a deductive system of $s$, then $x$ is a theorem of $s$ by $d$ just in case $\langle x\rangle$ is a proof in $s$ by $d$ and $x$ is a formula of $s$.

Given a symbol sequence $s$, a particular deductive system of $s L_{s}$ can be identified with $\log i c$ for $s$. The next three definitions characterize $L_{s}$ for any symbol sequence $s$.

Definition 36. If $s$ is a symbol sequence, then

(1) $\mathrm{T}_{s}=\left\langle N_{s}<\mathbf{E}_{s}<\mathbf{1}_{s}\right.$ the $1^{\text {th }}$ variable of $s<N_{s}<\mathbf{I}_{s}$ the $1^{\text {th }}$ variable of $s$ the $1^{\text {th }}$ variable of $s \gg \gg \gg$

(2) if $t$ is a finite sequence and the range of $t$ is included in the set of all lines of $s$, then

(a) for any positive integer $m$, if $m$ is not greater than the number of members of the set of all $k$ in the domain of $t$ such that $t(k)$ is a formula 
of $s$, then the $m^{\text {th }}$ formula of $s$ in $t=$ the formula of $s f$ such that, for some $l$ in the domain of $t, f=t(l)$ and the number of members of the set of all $k$ in the domain of $t$ such that $k$ is smaller than $l$ and $t(k)$ is a formula of $s$ $=m-1$

(b) the $O^{\text {th }}$ conjunction for $t$ of $s=\mathrm{T}_{s}$

(c) if there is a formula of $s$ in the range of $t$, then the $1^{\text {th }}$ conjunction for $t$ of $s=$ the $1^{\text {th }}$ formula of $s$ in $t$

(d) for any positive integer $m$, if $m$ is greater than 1 and $m$ is not greater than the number of members of the set of all $k$ in the domain of $t$ such that $t(k)$ is a formula of $s$, then the $m^{\text {th }}$ conjunction for $t$ of $s=<$ the $m-1^{\text {th }}$ conjunction for $t$ of $s \wedge_{s}$ the $m^{\text {th }}$ formula of $s$ in $t>$

(e) the conjunction for $t$ of $s=$ the (the number of members of the set of all $k$ in the domain of $t$ such that $t(k)$ is a formula of $s)^{\text {th }}$ conjunction for $t$ of $s$.

Definition 37. If $s$ is a symbol sequence, then

(1) $\mathbf{A}_{s}=$ the assumption rule of $s a$ such that, for any $t$ in the domain of $s, a(t)=$ the set of all $x$ such that, for some formulas of $s f$ and $g, t=$ $\left\langle\right.$ show $_{s}\langle f \rightarrow s g\rangle$ and $x=f$

(2) $\mathrm{EX}_{s_{1}}=$ the inference rule of $s r$ such that, for any $t$ in the domain of $r, r(t)=$ the set of all $x$ such that, for some positive integer $m, m$-place operation symbol of $s b, m$-term sequence $u$ whose range is included in the set of all terms of $s$, and $c$ in the range of $u,\left\langle E_{s}\langle b\rangle u\right\rangle$ is in the range of $t$ and $x=\left\langle\mathrm{E}_{s} c\right\rangle$

(3) $\mathrm{EX}_{s 2}=$ the inference rule of $s r$ such that, for any $t$ in the domain of $r, r(t)=$ the set of all $x$ such that, for some positive integer $m, m$-place predicate of $s b, m$-term sequence $u$ whose range is included in the set of all terms of $s$, and $c$ in the range of $u,\langle b\rangle u$ is in the range of $t$ and $x=$ $\left\langle\mathrm{E}_{s} c\right\rangle$

(4) $\mathrm{EX}_{s 3}=$ the inference rule of $s r$ such that, for any $t$ in the domain of $r, r(t)=$ the set of all $x$ such that, for some formulas of $s f$ and $g$ and variables of $s u, v$, and $\left.w, \ll \vee_{s} u f\right\rangle v_{s}\left\langle v_{s}\left\langle\wedge_{s} v g\right\rangle\right\rangle$ is in the range of $t$ and $x=\left\langle\mathbf{E}_{s} w\right\rangle$

(5) $\mathrm{EX}_{s_{4}}=$ the inference rule of $s r$ such that, for any $t$ in the domain of $r, r(t)=$ the set of all $x$ such that, for some distinct variables of $s v$ and $w$ and formula of $s f$ in which $v$ is not free, $\left\langle\vee_{s} v\left\langle\wedge_{s} w\left\langle f \leftrightarrow{ }_{s}\left\langle\mathbf{I}_{s} w v\right\rangle\right\rangle\right\rangle\right\rangle$ is in the range of $t$ and $x=\left\langle\mathbf{E}_{s}\left\langle\boldsymbol{1}_{s} w f\right\rangle\right\rangle$

(6) $\mathrm{TI}_{s}=$ the inference rule of $s r$ such that, for any $t$ in the domain of $r, r(t)=$ the set of all formulas of $s f$ such that $<$ the conjunction for $t$ of $s \rightarrow{ }_{s} f>$ is a tautology of $s$

(7) $\mathrm{UI}_{s}=$ the inference rule of $s r$ such that, for any $t$ in the domain of $r, r(t)=$ the set of all $x$ such that, for some variable of $s v$, formula of $s f$, and term of $s a$, both $\left\langle\Lambda_{s} v f\right\rangle$ and $\left\langle\mathrm{E}_{s} a\right\rangle$ are in the range of $t$ and $x=$ the result of properly substituting $a$ for $v$ in $f$ in $s$

(8) $\mathrm{EG}_{s}=$ the inference rule of $s r$ such that, for any $t$ in the domain of $r, r(t)=$ the set of all $x$ such that, for some variable of $s v$, formula of $s f$, and term of $s a$, both the result of properly substituting $a$ for $v$ in $f$ in $s$ and $\left\langle\mathrm{E}_{s} a\right\rangle$ are in the range of $t$ and $x=\left\langle\mathrm{V}_{s}\right.$ vf $\rangle$ 
(9) $\mathrm{EI}_{s}=$ the inference rule of $s r$ such that, for any $t$ in the domain of $r, r(t)=$ the set of all $x$ such that, for some variables of $s v$ and $w$ and formula of $s f,\left\langle\vee_{s} v f\right\rangle$ is in the range of $t$, there is no $g$ in the range of $t$ such that $w$ occurs in $g$, and $x=$ the result of properly substituting $w$ for $v$ in $f$ in $s$

(10) ID $_{s 1}=$ the inference rule of $s r$ such that, for any $t$ in the domain of $r, r(t)=$ the set of all $x$ such that, for some terms of $s a$ and $b$ and formula of $s f$, both $\left\langle\left\langle\mathbf{I}_{s} a b\right\rangle v_{s}\left\langle\left\langle N_{s}\left\langle\mathrm{E}_{s} a\right\rangle{\wedge_{s}}_{s}\left\langle N_{s}\left\langle\mathrm{E}_{s} b \gg \gg\right\rangle\right.\right.\right.\right.$ and the result of properly substituting $a$ for $b$ in $f$ in $s$ are in the range of $t$ and $x=f$

(11) ID $_{s 2}=$ the inference rule of $s r$ such that, for any $t$ in domain of $r$, $r(t)=$ the set of all $x$ such that, for some term of $s a,\left\langle\mathrm{E}_{s} a\right\rangle$ is in the range of $t$ and $x=\left\langle\mathbf{I}_{s} a a\right\rangle$

(12) $\mathrm{D}_{s_{1}}=$ the inference rule of $s r$ such that, for any $t$ in the domain of $r, r(t)=$ the set of all $x$ such that, for some distinct variables of $s v$ and $w$ and formula of $s f$ in which $v$ is not free, $\left\langle\mathrm{V}_{s} v\left\langle\wedge_{s} w\left\langle f \leftrightarrow{ }_{s}\left\langle\mathbf{I}_{s} w v\right\rangle \gg\right\rangle\right.\right.$ is in the range of $b$ and $x=$ the result of properly substituting $\left\langle\boldsymbol{1}_{s} w f\right\rangle$ for $w$ in $f$ in $s$

(13) $\mathrm{D}_{s 2}=$ the inference rule of $s r$ such that, for any $t$ in the domain of $r, r(t)=$ the set of all $x$ such that, for some distinct variables of $s v$ and $w$ and formula of $s f$ in which $v$ is not free, $\left\langle\mathbf{E}_{s}\left\langle\mathfrak{1}_{s} w f\right\rangle\right\rangle$ is in the range of $t$ and $x=\left\langle\vee_{s} v\left\langle\wedge_{s} w\left\langle f \leftrightarrow{ }_{s}\left\langle\mathrm{I}_{s} w v \gg\right\rangle\right\rangle\right.\right.$

(14) $\mathrm{DP}_{s}=$ the proof method of $s p$ such that, for any $t$ in the domain of $p, p(t)=$ the set of all $x$ such that, for some formula of $s f$ and $m$ in the domain of $t$, the following conditions are satisfied:

(a) $t(m)=\langle$ showis $f\rangle$

(b) there is no $n$ in the domain of $t$ greater than $m$ such that, for some formula of $s g, t(n)=\left\langle\right.$ show $\left._{s} g\right\rangle$

$t(n)=f$

(c) there is an $n$ in the domain of $t$ greater than $m$ such that

(d) $x=$ (the restriction of $t$ to the set of all $l$ in the domain of $t$ such that $l$ is smaller than $m)\left(\begin{array}{c}m \\ f\end{array}\right)$

(15) $\mathrm{UP}_{s}=$ the proof method of $s p$ such that, for any $t$ in the domain of $p, p(t)=$ the set of all $x$ such that, for some variable of $s v$, formula of $s f$, and $m$ in the domain of $t$, the following conditions are satisfied:

(a) for any $l$ in the domain of $t$, if $l$ is smaller than $m$ and $t(l)$ is a formula of $s$, then $v$ is not free in $t(l)$

(b) $t(m)=\left\langle\right.$ show $\left._{s}\left\langle\Lambda_{s} v f\right\rangle\right\rangle$

(c) there is no $n$ in the domain of $t$ greater than $m$ such that, for some formula of $s, t(n)=\left\langle\right.$ show $\left._{s} g\right\rangle$

$t(n)=f$

(d) there is an $n$ in the domain of $t$ greater than $m$ such that

(e) $x=$ (the restriction of $t$ to the set of all $l$ in the domain of $t$ such that $l$ is smaller than $m)\left(\begin{array}{l}m \\ <\wedge s\end{array} \wedge_{f}>\right)$.

Given a symbol sequence $s$ and formulas of $s f$ and $g, \mathbf{A}_{s}$ is, of course, the rule which allows one to assume $f$ in order to prove $f \rightarrow g$. The four $E_{s}$ inference rules are existence rules in the sense that they allow us to infer the existence statements form their conditions. $\mathbf{T I}_{s}$ and $\mathbf{E I}_{s}$ are the inference rules of tautological implication and existential instantiation 
respectively while $\mathrm{UI}_{s}$ and EG $s$ are variants for a logic applicable in the empty set of the inference rules of universal instantiation and existential generalization respectively. ID Is $_{s 1}$ is a form of the principle of the indiscernibility of identicals which, just like the other rules of transformation of definition 37 which have to do with terms of $s$, is not applicable within the scope of $O$-place variable binders of $s$ and according to which non-existents are extensionally indiscernible. ID $_{s_{2}}$ is a form of the principle of self identity which is only applicable to a term of $s t$ if $E t$ can be established. $D_{s_{1}}$ expresses the fact that the so and so is so and so if there is exactly one so and so and $D_{s 2}$ expresses the fact that there is exactly one so and so if the so and so exists. $\mathrm{DP}_{s}$ is, of course, the method of completing a proof by producing the formula of $s$ to be proven and UP ${ }_{s}$ is the method of completing the proof of a universal generalization by producing the formula of $s$ inside of the quantifier phrase without loss of generality.

Notice that some of the rules of transformation of definition 37 (particularly $\mathrm{TI}_{s}$ and $\mathrm{ID}_{s_{1}}$ ) are partially redundant.

Definition 38. If $s$ is a symbol sequence, then $L_{s}=$ the deductive system of $s d$ satisfying the following conditions:

(1) $d(1)=$ the empty set

(2) $d(2)=\left\{\mathbf{A}_{s}\right\}$

(3) $d(3)=$ the set of all $x$ satisfying one of the following conditions:

(a) for some positive integer $m$ not greater than 4, $x=\mathbf{E X}_{s m}$

(b) either $x=\mathbf{T I}_{s}$ or $x=\mathbf{U I}_{s}$ or $x=\mathbf{E G}_{s}$ or $x=\mathbf{E I}_{s}$

(c) for some positive integer $m$ not greater than 2 , either $x=$ $\mathrm{ID}_{s m}$ or $x=\mathrm{D}_{s m}$

(4) $d(4)=\left\{\mathrm{DP}_{s}\right.$ UP $\left._{s}\right\}$.

In other words, given a symbol sequence $s, L_{s}$ is the axiomless deductive system of $s d$ whose rules of transformation are just those defined in definition 37.

Notice that, for any symbol sequence $s$, there are various deductive systems of $s d$ whose theorems are those of $\mathrm{L}_{s}$ although $d(1), d(2)$, and $d(3)$ are empty, i.e., whose only rules of transformation are proof methods. A simple deductive system of this kind is the one whose only proof method allows one to complete a proof whenever the formula of $s$ to be proven is a theorem of $s$ by $L_{s}$ (however that is determined). On the other hand, there are no deductive systems of $s d$ with $d(4)$ empty such that, for some $f, f$ is a theorem of $s$ by $d$; in other words, a deductive system with no proof methods produces no theorems.

\section{THE SOUNDNESS OF $L_{s}$}

In this section, it is shown that, for any symbol sequence $s$, any theorem of $s$ by $L_{s}$ is valid with respect to $s$. Some auxiliary concepts will be defined so as to make the proof of this and later facts more clear.

Definition 39.

(1) if either $p$ is a finite sequence or $p$ is a denumerably infinite sequence, then 
(a) if $m$ is a natural number, then $p$ cut off at the $m^{\text {th }}$ term $=$ the restriction of $p$ to the set of all $l$ in the domain of $p$ such that $l$ is smaller than $m$

(b) if $p$ is a finite sequence and $p$ is not empty, then

(1) the last term of $p=p$ (the greatest member of the domain of $p)$

(2) $p$ cut off at the last term $=p$ cut off at (the greatest member of the domain of $p)^{\text {th }}$ term

(3) if $s$ is a symbol sequence, then

(a) $p$ consists of a show line of $s$ just in case, for some formula of $s f, p=\left\langle\right.$ show $_{s} f \gg$

(b) $q$ is obtainable from $p$ by adding a show line of $s$ just in case, for some formula of $s, q=\overparen{p} \ll$ shows $_{s} f \gg$

(c) $q$ is obtainable from $p$ by adding an assumption of $s$ just in case, for some formulas of $s f$ and $g$, the last term of $p=\left\langle\right.$ show $_{s}$ $\left\langle f \rightarrow{ }_{s} g\right\rangle$ and $q=\widehat{p}\langle f\rangle$

(d) $q$ is obtainable from $p$ by an existential instantiation of $s$ just in case, for some $v, w$, and $f, v$ and $w$ are variables of $s, f$ is a formula of $s,\left\langle\mathrm{~V}_{s} v f\right\rangle$ is in the range of $p$, there is no $g$ in the range of $p$ such that $w$ occurs in $g$, and $q=\widetilde{p}<$ the result of properly substituting $w$ for $v$ in $f$ in $s>$

(e) $q$ is obtainable from $p$ by an inference rule of $s$ just in case, for some $r$ and $f, r$ is in $L_{s}(3), p$ is in the domain of $r, f$ is in $r(p)$, and $q=\widehat{p}\langle f>$

(f) $q$ is obtainable from $p$ by an inference rule of $s$ other than existential instantiation just in case $q$ is obtainable from $p$ by an inference rule of $s$ and $q$ is not obtainable from $p$ by an existential instantiation of $s$

(g) $q$ is obtainable from $p$ by a direct proof in $s$ just in case, for some formula of $s f$ and $m$ in the domain of $p$, the following conditions are satisfied:

(1) $p(m)=\left\langle\right.$ show $\left._{s} f\right\rangle$

(2) there is no $n$ in the domain of $p$ greater than $m$ such that, for some formula of $s g, p(n)=\left\langle\right.$ shows $\left._{s}\right\rangle$

(3) there is an $n$ in the domain of $p$ greater than $m$ such that $p(n)=f$

(4) $q=\left(p\right.$ cut off at the $m^{\text {th }}$ term $\widetilde{\langle}\langle f\rangle$

(h) $q$ is obtainable from $p$ by a universal proof in $s$ just in case, for some variable of $s v$, formula of $s f$, and $m$ in the domain of $p$, the following conditions are satisfied:

(1) for any $l$ in the domain of $p$, if $l$ is smaller than $m$ and $p(l)$ is a formula of $s$, then $v$ is not free in $p(l)$

(2) $p(m)=\left\langle\right.$ show $_{s}\left\langle\Lambda_{s}\right.$ vf $\rangle$

(3) there is no $n$ in the domain of $p$ greater than $m$ such that, for some formula of $s g, p(n)=\left\langle\right.$ show $\left._{s} g\right\rangle$

(4) there is an $n$ in the domain of $p$ greater than $m$ such that $p(n)=f$

(5) $q=\left(p\right.$ cut off at the $m^{\text {th }}$ term $\widehat{\ll} \ll \wedge_{s} v f \gg$ 
(i) $q$ is obtainable from $p$ in $s$ just in case either $q$ is obtainable from $p$ by adding a show line of $s$ or $q$ is obtainable from $p$ by adding an assumption of $s$ or $q$ is obtainable from $p$ by an inference rule of $s$ or $q$ is obtainable from $p$ by a direct proof in $s$ or $q$ is obtainable from $p$ by a universal proof in $s$.

(2) if $s$ is a symbol sequence, then $t$ is a proof sequence in $s$ just in case $t$ satisfies the following conditions:

(a) either $t$ is not empty and $t$ is a finite sequence or $t$ is a denumerably infinite sequence

(b) $t$ (1) consists of a show line of $s$

(c) for any $m$ in the domain of $t$, if $m$ is greater than 1 , then $t(m)$ is obtainable from $t(m-1)$ in $s$.

Theorem 1. If $s$ is a symbol sequence, $t$ is a proof sequence in $s$, and $m$ is in the domain of $t$, then, for any $l$ in the domain of $t$, if $l$ is greater than $1, l$ is not greater than $m$, and either $t(l)$ is obtainable from $t(l-1)$ by a direct proof in $s$ or $t(l)$ is obtainable from $t(l-1)$ by a universal proof in $s$, then $<$ the conjunction for $\left(t(l)\right.$ cut off at the last term) of $s \rightarrow_{s}$ the last term of $t(l)>$ is valid with respect to $s$.

Assume that $s$ is a symbol sequence and that $t$ is a proof sequence in $s$. For any positive integer $m$ and any $l$, let Alm hold just in case $l$ is in the domain of $t, l$ is greater than $1, l$ is not greater than $m$, and either $t(l)$ is obtainable from $t(l-1)$ by a direct proof in $s$ or $t(l)$ is obtainable from $t(l-1)$ by a universal proof in $s$. Also, for any $l$ in the domain of $t$, let $\mathrm{C} l$ hold just in case the conjunction for $(t(l)$ cut off at the last term) of $s \rightarrow$ the last term of $t(l)$ is valid with respect to $s$. Let $x=$ the set of all positive integers $m$ such that, if $m$ is in the domain of $t$, then, for any $l$, if Alm, then $\mathrm{Cl} . \quad 1$ is in $x$ since there is no $l$ in the domain of $t$ such that $l$ is both greater and not greater than 1 . To establish the theorem, we must now show that, for any positive integer $m$, if $m$ is in $x$, then $m+1$ is in $x$. Assume then that $m$ is a positive integer, that $m$ is in $x$, that $m+1$ is in the domain of $t$, and that Alm +1 . It follows that $m$ is in the domain of $t$ and so that, for any $l, \mathrm{Cl}$ if $\mathrm{Alm}$. To establish the theorem, it is by definition 39 sufficient to show that

(1) if $t(m+1)$ is obtainable from $t(m)$ by adding a show line of $s$, then $\mathrm{Cl}$

(2) if $t(m+1)$ is obtainable from $t(m)$ by adding an assumption of $s$, then $\mathrm{Cl}$ then $\mathrm{Cl}$

(3) If $t(m+1)$ is obtainable from $t(m)$ by an inference rule of $s$,

(4) if $t(m+1)$ is obtainable from $t(m)$ by a direct proof in $s$, then $\mathrm{Cl}$

(5) if $t(m+1)$ is obtainable from $t(m)$ by a universal proof in $s$, then C $l$.

To show (1), assume that $t(m+1)$ is obtainable from $t(m)$ by adding a show line of $s$. Since $t(l)$ is obtainable from $t(l-1)$ by either a direct or a universal proof in $s, l \neq m+1$ by definition 39 and so $l$ is not greater than $m$; hence, Alm and so $\mathrm{C} l$. 
By reasoning as for (1), it can be shown that both (2) and (3) hold.

To show (4), assume that $t(m+1)$ is obtainable from $t(m)$ by a direct proof in $s$. By definition 39, it follows that, for some formula of $s f$ and $k$ in the domain of $t(m),(t(m))(k)=$ show $f$, there is no $n$ in the domain of $t(m)$ greater than $k$ such that, for some formula of $s g,(t(m))(n)=$ show $g$, there is an $n$ in the domain of $t(m)$ greater than $k$ such that $(t(m))(n)=f$, and $t(m+1)=\left(t(m)\right.$ cut off at the $k^{\text {ith }}$ term $\widetilde{\langle}\langle f$. If $l \neq m+1$, then $c l$ by reasoning as in the later portion of the proof for (1). Assume then that $l=$ $m+1$. We shall show that

(a) the conjunction for $\left(t(m)\right.$ cut off at the $n^{\text {th }}$ term) of $s \rightarrow f$ is valid with respect to $s$.

Let $a=t(m)$ cut off at the $n^{\text {th }}$ term. It is not possible that $\overparen{a<f>\text { is }}$ obtainable from $a$ by adding either a show line of $s$ or an assumption of $s$ since then either there is an $n$ greater than $k$ in the domain of $t(m)$ and a formula of $s g$ such that $(t(m))(n)=$ show $g$ or there are formulas of $s g$ and $h$ such that $(t(m))(k)=$ show $f=$ show $g \rightarrow h$ and $f=g$. Also, if $\overparen{a<f>\text { is }}$ obtainable from $a$ by an inference rule of $s$ other than existential instantiation, then we obviously have (a). Assume then that $\widetilde{a}\langle f\rangle$ is obtainable from $a$ by an existential instantiation of $s$; hence, by definition 39 , for some $v, w$, and $g, v$ and $w$ are variables of $s, g$ is a formula of $s, V v g$ is in the range of $a$, there is no $h$ in the range of $a$ such that $w$ occurs in $h$, and $f=$ the result of properly substituting $w$ for $v$ in $g$ in $s$. Then, since $a(k)=$ show $f$ and $w$ does not occur in $a(k), v$ cannot be free in $g$. But, for any variable of $s v$ and formula of $s g$, if $v$ is not free in $g$, then $\vee v g \rightarrow g$ is valid with respect to $s$ and, for any variable of $s w$, the result of properly substituting $w$ for $v$ in $g$ in $s=g$. Hence, the conjunction for $a$ of $s \rightarrow f$ is valid with respect to $s$ and so (a) holds.

Assume finally that, for some $j$ in the domain of $t$ smaller than $m$, $\widetilde{a<f>}=t(j+1)$ and $\overparen{a<f>}$ is obtainable from $t(j)$ either by a direct proof in $s$ or by a universal proof in $s$. Since $j+1$ is greater than 1 and not greater than $m$, we then have $\mathbf{A} j+1 m$ and so $\mathbf{C} j+1$. Hence, since $t(j+1)$ cut of $\mathrm{f}$ at the last term $=a$ and the last term of $t(j+1)=f$, we again have (a). Thus, by definition 39 , the ways in which $\overparen{a}\langle f\rangle$ is obtainable from its predecessor in $t$ are exhausted and (a) holds.

We now show by induction that

(b) for any natural number $z$, if $z$ is not greater than $(n-1)-k$, then the conjunction for $\left(t(m)\right.$ cut off at the $n-z^{\text {th }}$ term) of $s \rightarrow f$ is valid with respect to $s$.

By (a), (b) holds for 0 . For any natural number $z$ not greater than $(n-1)-k$, let $\mathrm{K} z=$ the conjunction for $\left(t(m)\right.$ cut off at the $n-z^{\text {th }}$ term) of $s$. Assume that $z$ is a natural number, that $\mathbf{K} z \rightarrow f$ is valid with respect to $s$ if $z$ is not greater than $(n-1)-k$, and that $z+1$ is not greater than $(n-1)-k$. It follows that $z$ is not greater than $(n-1)-k$ and so that $\mathrm{K} z \rightarrow f$ is valid with respect to $s$. Let $b=t(m)$ cut off at the $n-(z+1)^{\text {th }}$ term and let $c=t(m)$ cut off at the $n-z^{\text {th }}$ term. We must show that $\mathbf{K} z+1 \rightarrow f$ is valid with respect to $s$.

It is not possible that $c$ is obtainable from $b$ by adding a show line of $s$ 
since then there is an $n$ in the domain of $t(m)$ greater than $k$ such that, for some formula of $s g,(t(m))(n)=$ show $g$.

Assume that $c$ is obtainable from by adding an assumption of $s$; hence, for some formulas of $s g$ and $h,(t(m))(n-(z+2))=$ show $g \rightarrow h$ and $(t(m))(n-(z+1))=g$. Since there is no $n$ in the domain of $t(m)$ greater than $k$ such that, for some formula of $s i,(t(m))(n)=$ show $i$, and since $n-(z+2)$ is not smaller than $k, n-(z+2)=k$ and so $(t(m))(n)=g \rightarrow h$. Then, since $\mathrm{K} z=$ the $1^{\text {th }}$ formula of $s$ in $c=g$ if there is no formula of $s$ in the range of $b, \mathbf{K} z=\mathbf{K} z+1 \wedge g$ if there is a formula of $s$ in the range of $b, k \rightarrow\langle g \rightarrow h\rangle$ is valid with respect to $s$ if either $g \rightarrow\langle g \rightarrow h\rangle$ or $k \wedge g \rightarrow\langle g \rightarrow h\rangle$ is valid with respect to $s$ for any formulas of $s k, g$, and $h$, and $\mathrm{K} z \rightarrow f$ is valid with respect to $s, \mathbf{K} z+1 \rightarrow f$ is valid with respect to $s$.

Obviously, if $c$ is obtainable from $b$ by an inference rule of $s$ other than existential instantiation, then $\mathbf{K} z+1 \rightarrow \mathbf{K} z$ is valid with respect to $s$ and so $\mathrm{K} z+1 \rightarrow f$ is valid with respect to $s$. Assume then that $c$ is obtainable from $b$ by an existential instantiation of $s$; hence, by definition 39, for some $v, w$, and $g, v$ and $w$ are variables of $s, g$ is a formula of $s, \vee v g$ is in the range of $b$, there is no $h$ in the range of $b$ such that $w$ occurs in $h$, and $(t(m))(n-(z+1))=$ the result of properly substituting $w$ for $v$ in $g$ and $s$. Then, since $w$ is not free in either $\mathbf{K} z+1$ or $g$ or $f$ and since, for any formulas of $s f, g$, and $k$ and variables of $s v$ and $w$, if $w$ is not free in either $f$ or $g$ or $k$ and both $k \wedge$ the result of properly substituting $w$ for $v$ in $g$ in $s \rightarrow f$ and $k \rightarrow \vee v g$ are valid with respect to $s$, then $k \rightarrow f$ is valid with respect to $s, \mathrm{~K} z+1 \rightarrow f$ is valid with respect to $s$.

Assume finally that, for some $j$ in the domain of $t$ smaller than $m, c=$ $t(j+1)$ and $c$ is obtainable from $t(j)$ either by a direct proof in $s$ or by a universal proof in $s$. Since $j+1$ is greater than 1 and not greater than $m$, we then have $\mathrm{A} j+1 \mathrm{~m}$ and so $\mathrm{C} j+1$, i.e., the conjunction for ( $c$ cut off at the last term) of $s \rightarrow$ the last term of $c$ is valid with respect to $s$. Since $c$ cut off at the last term $=t(m)$ cut off at the $n-(z+1)^{\text {th }}$ term and the last term of $c=(t(m))(n-(z+1)), \mathbf{K} z+1 \rightarrow(t(m))(n-(z+1))$ is valid with respect to $s$. Also, since $c$ is obtainable from $t(j)$ either by a direct proof in $s$ or by a universal proof in $s,(t(m))(n-(z+1))$ is a formula of $s$. Hence, since $\mathrm{K} z=$ the $1^{\text {th }}$ formula of $s$ in $c=(t(m))(n-(z+1))$ if there is no formula of $s$ in the range of $b$ and $\mathbf{K} z=\mathbf{K} z+1 \wedge(t(m))(n-(z+1))$ if there is a formula of $s$ in the range of $b, \mathrm{~K} z+1 \rightarrow \mathrm{K} z$ is valid with respect to $s$. Since $\mathrm{K} z \rightarrow f$ is valid with respect to $s$, it follows that $\mathrm{K} z+1 \rightarrow f$ is valid with respect to $s$. Thus, by definition 39 , the ways in which $c$ is obtainable from its predecessor in $t$ are exhausted and (b) holds.

If we instantiate the $z$ in (b) to $(n-1)-k$, then we obtain that the conjunction for $\left(t(m)\right.$ cut off at the $k+1^{\text {th }}$ term) of $s \rightarrow f$ is valid with respect to $s$. But, since $(t(m))(k)=$ show $f$, the conjunction for $(t(m)$ cut off at the $k+1^{\text {th }}$ term) of $s=$ the conjunction for $\left(t(m)\right.$ cut off at the $k^{\text {th }}$ term) of $s$. Also, $t(m)$ cut off at the $k^{\text {th }}$ term $=t(m+1)$ cut off at the last term and $f=$ the last term of $t(m+1)$. Hence, the conjunction for $(t(m+1)$ cut off at the last term) of $s \rightarrow$ the last term of $t(m+1)$ is valid with respect to $s$. Since $l=m+1, \mathrm{Cl}$ and so (4) holds. 
To show that (5) holds, assume that $t(m+1)$ is obtainable from $t(m)$ by a universal proof in $s$. By definition 39 , it follows that, for some variable of $s w$, formula of $s f$, and $k$ in the domain of $t(m), w$ is not free in $(t(m))(j)$ if $j$ is in the domain of $t(m), j$ is smaller than $k$, and $(t(m))(j)$ is a formula of $s$ for any $j,(t(m))(k)=$ show $\wedge w f$, there is no $n$ in the domain of $t(m)$ greater than $k$ such that, for some formula of $s g,(t(m))(n)=$ show $g$, the re is an $n$ in the domain of $t(m)$ greater than $k$ such that $(t(m))(n)=f$, and $t(m+1)=\left(t(m)\right.$ cut off at the $k^{\text {th }}$ term $\widetilde{<} \wedge w f>$. If $l \neq m+1$, then $\mathrm{Cl}$ by reasoning as in the later portion of the proof for (1). Assume then that $l=m+1$. It can be shown that

(a) the conjunction for $\left(t(m)\right.$ cut off at the $n^{\text {th }}$ term) of $s \rightarrow f$ is valid with respect to $s$.

The reasoning is the same as that for (a) under (4), but with $(t(m))(k)$ $=$ show $\wedge w f$.

It can also be shown that

(b) for any natural number $z$, if $z$ is not greater than $(n-1)-k$, then the conjunction for $\left(t(m)\right.$ cut off at the $n-z^{\text {th }}$ term) of $s \rightarrow f$ is valid with respect to $s$.

The reasoning is the same as for (b) under (4), but with the case of $c$ being obtainable from $b$ by adding an assumption of $s$ impossible since $(t(m))(k)=$ show $\wedge w f$.

By reasoning with (b) as under (4), it can be shown that the conjunction for $\left(t(m)\right.$ cut off at the $k^{\text {th }}$ term) of $s \rightarrow f$ is valid with respect to $s$. Since $w$ is not free in the conjunction for $\left(t(m)\right.$ cut off at the $k^{\text {th }}$ term) of $s$, since $t(m)$ cut off at the $k^{\text {th }}$ term $=t(m+1)$ cut off at the last term, since $\wedge w f=$ the last term of $t(m+1)$, and since $c \rightarrow \wedge w f$ is valid with respect to $s$ if $c \rightarrow f$ is for any variable of $s w$ and formulas of $s c$ and $f$ such that $w$ is not free in $c$, the conjunction for $(t(m+1)$ cut off at the last term) of $s \rightarrow$ the last term of $t(m+1)$ is valid with respect to $s$. Since $l=m+1, \mathrm{Cl}$. Hence, (5) holds and so the theorem holds.

Theorem 2. If $s$ is a symbol sequence and $p$ is a proof in $s$ by $L_{s}$, then there is a finite sequence $t$ such that $t$ is a proof sequence in $s$ and the last term of $t=p$.

Assume that $s$ is a symbol sequence and let $x=$ the set of all $p$ such that $p$ is a proof in $s$ by $L_{s}$ and there is a finite proof sequence in $s t$ such that the last term of $t=p$. By definitions $34,38,37$, and 39 , it is sufficient to show that, for any $p$ in $q$,

(1) if $p$ consists of a show line of $s$, then $p$ is in $x$

(2) if $p$ is in $x$ and $q$ is obtainable from $p$ in $s$, then $q$ is in $x$.

Assume that $p$ consists of a show line of $s$ and let $u=\langle p\rangle$. By definition $39, u$ is a proof sequence in $s$ and the last term of $u=p$. Hence, $p$ is in $x$.

Assume finally that $p$ is in $x$ and that $q$ is obtainable from $p$ in $s$. Since $p$ is in $x$, there is a finite proof sequence in $s r$ such that the last term of $r=p$. Let $u=\widetilde{r}\langle q\rangle$. Because $q$ is obtainable from $p$ in $s, u$ is a proof sequence in s. Also, the last term of $u=q$. Hence, $q$ is in $x$ and the theorem holds. 
Theorem 3. If $s$ is a symbol sequence and $f$ is a theorem of $s$ by $L_{s}$, then $f$ is valid with respect to $s$.

Assume the antecedent. By definition $35,\langle f\rangle$ is a proof in $s$ by $L_{s}$. Hence, by definitions $34,38,37$, and 39 , there is a proof in $s$ by $L_{s} p$ such that either $\langle f\rangle$ is obtainable from $p$ by a direct proof in $s$ or $\langle f\rangle$ is obtainable from $p$ by a universal proof in $s$. By theorem 2, there is a finite proof sequence in $s r$ such that the last term of $r=p$. Let $j=$ the greatest member of the domain of $r$ and let $t=\widetilde{r}\langle f\rangle$. Since $j+1$ is in the domain of $t$, since $j+1$ is greater than 1 , since $j+1$ is not greater than $j+1$, since $t(j)=p$, and since $t(j+1)=\langle f\rangle$, we obtain from theorem 1 that the conjunction for $(t(j+1)$ cut off at the last term) of $s \rightarrow$ the last term of $t(j+1)$ is valid with respect to $s$ by letting $t=t$, letting $m=j+1$, and instantiating $l$ to $j+1$. Since $t(j+1)=\langle f\rangle$, since $\langle f\rangle$ cut off at the last term is empty, and since the last term of $\langle f\rangle=f$, it follows by definition 36 that $\mathbf{T}_{s} \rightarrow f$ is valid with respect to $s$. Then, since $\mathbf{T}_{s}$ is valid with respect to $s, f$ is valid with respect to $s$.

\section{THE SEMANTIC COMPLETENESS OF $L_{s}$}

In this section, it is shown that, for any symbol sequence $s$, any formula of $s$ valid with respect to $s$ is a theorem of $s$ by $L_{s}$. Some additional auxiliary concepts will be defined.

Definition 40. If $s$ is a symbol sequence, then

(1) $t$ is an enumerator of the formulas of $s$ just in case $t$ is a denumerably infinite sequence, the range of $t=$ the set of all formulas of $s$, and, for any $i$ and $j$ in the domain of $t$, if $i \neq j$, then $t(i) \neq t(j)$

(2) if $m$ is a positive integer and $t$ is an enumerator of the formulas of $s$, then the $m^{\text {th }}$ existential generalization of $s$ in $t=$ the formula of $s f$ such that, for some $v$ and $g, f=\left\langle\mathrm{V}_{s} v g\right\rangle$ and, for any positive integer $n$, if $t(n)$ $=f$, then the number of members of the set of all positive integers $l$ such $l$ is smaller than $n$ and, for some $w$ and $h, t(l)=\left\langle\mathrm{V}_{s} w h\right\rangle=m-1$

(3) if either $t$ is a term of $s$ or $t$ is a formula of $s$, then

(a) if $m$ is a positive integer, then

(1) if $m$ is not greater than the number of members of the set of all variables of $s v$ such that $v$ is free in $t$, then the $m^{\text {th }}$ variable of $s$ free in $t=$ the $v$ such that, for some positive integer $l, v=$ the $l^{\text {th }}$ variable of $s, v$ is free in $t$, and the number of members of the set of all positive integers $k$ smaller than $l$ such that the $k^{\text {th }}$ variable of $s$ is free in $t=m-1$

(2) if $m$ is not greater than the number of members of the set of all individual constants of $s c$ such that $c$ occurs in $t$, then

(a) the $m^{\text {th }}$ individual constant of $s$ in $t=$ the $c$ such that for some positive integer $l, c=$ the $l^{\text {th }}$ individual constant of $s, c$ occurs in $t$, and the number of members of the set of all positive integers $k$ smaller than $l$ such that the $k^{\text {th }}$ individual constant of $s$ occurs in $t=m-1$

(b) the $m^{\text {th }}$ correlated individual constant of $s$ for $t=$ the $x$ such that, for some positive integer $l$, the $m^{\text {th }}$ individual constant of $s$ in $t=$ the $l^{\text {th }}$ individual constant of $\mathrm{s}$ and $x=$ the $2 l^{\text {th }}$ individual constant of $s$ 
(b) the sequence of variables of $s$ free in $t=$ the $x$ satisfying the following conditions:

(1) $x$ is a function

(2) the domain of $x=$ the set of all positive integers $m$ such that $m$ is not greater than the number of members of the set of all variables of $s v$ such that $v$ is free in $t$

$s$ free in $t$

(3) for any $m$ in the domain of $x, x(m)=$ the $m^{\text {th }}$ variable of

(c) the sequence of individual constants of $s$ in $t=$ the $x$ satisfying the following conditions:

(1) $x$ is a function

(2) the domain of $x=$ the set of all positive integers $m$ such that $m$ is not greater than the number of members of the set of all individual constants of $s c$ such that $c$ occurs in $t$

(3) for any $m$ in the domain of $x, x(m)=$ the $m^{\text {th }}$ individual constant of $s$ in $t$

(d) the sequence of correlated individual constants of $s$ for $t=$ the $x$ satisfying the following conditions:

(1) $x$ is a function

(2) the domain of $x=$ the domain of the sequence of individual constants of $s$ in $t$

(3) for any $m$ in the domain of $x, x(m)=$ the $m^{\text {th }}$ correlated individual constant of $s$ for $t$

(e) if $u$ and $v$ are finite sequences, the domain of $u=$ the domain of $v$, and the union of the range of $u$ and the range of $v$ is included in the set all terms of $s$, then

(1) the $0^{\text {th }}$ result of properly substituting $v$ for $u$ in $t$ in $s=t$

(2) for any positive integer $m$, if $m$ is in the domain of $v$, then the $m^{\text {th }}$ result of properly substituting $v$ for $u$ in $t$ in $s=$ the result of properly substituting $v(m)$ for $u(m)$ in (the $m-1^{\text {th }}$ result of properly substituting $v$ for $u$ in $t$ in $s$ ) in $s$

(3) the result of properly substituting $v$ for $u$ in $t$ in $s=$ the (the $m$ such that either $v$ is empty and $m=0$ or $v$ is not empty and $m=$ the greatest member of the domain of $v)^{\text {th }}$ result of properly substituting $v$ for $u$ in $t$ in $s$

(f) the correlate of $t$ in $s=$ the $x$ such that, for some $v, v$ is a finite sequence, the domain of $v=$ the domain of the sequence of individual constants of $s$ in $t$, the range of $v$ is included in the set of all variables of $s$, there is no $l$ in the domain of $v$ such that either $v(l)$ occurs in $t$ or, for some $k$ in the domain of $v, k \neq l$ and $v(k)=v(l)$, and $x=$ the result of properly substituting (the sequence of correlated individual constants of $s$ for $t$ ) for $v$ in (the result of properly substituting $v$ for (the sequence of individual constants of $s$ in $t$ ) in $t$ in $s$ ) in $s$

(4) if $t$ is a proof sequence in $s$ and either $a$ and $b$ are terms of $s$ or ( $a$ and $b$ are finite sequences, the domain of $a=$ the domain of $b$, and the union of the range of $a$ and the range of $b$ is included in the set of all terms of $s$ ), then the result of properly substituting $b$ for $a$ in $t$ in $s=$ the $u$ satisfying the following conditions: 
(a) $u$ is a function

(b) the domain of $u=$ the domain of $t$

(c) for any $m$ in the domain of $u, u(m)=$ the $p$ satisfying the following conditions:

(1) $p$ is a finite sequence

(2) the domain of $p=$ the domain of $t(m)$

(3) for any $l$ in the domain of $p$, one of the following conditions is satisfied

(a) there is an $f$ such that $(t(m))(l)=\left\langle\right.$ show $\left._{s} f\right\rangle$ and $p(l)=\left\langle\right.$ show $_{s}$ the result of properly substituting $b$ for $a$ in $f$ in $\left.s\right\rangle$

(b) $(t(m))(l)$ is a formula of $s$ and $p(l)=$ the result of properly substituting $b$ for $a$ in $(t(m))(l)$ in $s$

(5) if $x$ is included in the set of all formulas of $s$, then

(a) the correlate of $x$ in $s=$ the set of all $y$ such that, for some $f$ in $x, y=$ the correlate of $f$ in $s$

(b) for any $f$, if $f$ is a formula of $s$, then $x$ implies $f$ in $s$ just in case, for some finite sequence whose range is included in $x t, t(i) \neq t(j)$ if $i \neq j$ for any $i$ and $j$ in the domain of $t$ and <the conjunction for $t$ of $s \rightarrow s f>$ is a theorem of $s$ by $L_{s}$

(c) $x$ is consistent in $s$ just in case, for some formula of $s f, x$ does not imply $f$ in $s$

(d) for any $t$ and $c$, if $t$ is an enumerator of the formulas of $s, c$ is a denumerably infinite sequence, the range of $c$ is included in the set of all individual constants of $s d$ such that $d$ is not free in $f$ for any $f$ in $x$, and $c(i) \neq c(j)$ if $i \neq j$ for any positive integers $i$ and $j$, then

(1) the $o^{\text {th }}$ instantial expansion of $x$ by $t$ and $c$ in $s=x$

(2) for any positive integer $m$, the $m^{\text {th }}$ instantial expansion of $x$ by $t$ and $c$ in $s=$ the union of the $m-1^{\text {th }}$ instantial expansion of $x$ by $t$ and $c$ in $s$ and $\left\{<\right.$ the $m^{\text {th }}$ existential generalization of $s$ in $t \rightarrow s$ the result of properly substituting the $d$ such that ( $d$ is not free in $f$ if $f$ is in the union of the $m-1^{\text {th }}$ instantial expansion of $x$ by $t$ and $c$ in $s$ and the $g$ such that, for some $v$, the $m^{\text {th }}$ existential generalization of $s$ in $\left.t=\left\langle\vee_{s} v g\right\rangle\right\}$ for any $f, d=c(l)$ for some positive integer $l$, and there is no positive integer $k$ smaller than $l$ such that $(c(k)$ is not free in $f$ if $f$ is in the union of the $m-1^{\text {th }}$ instantial expansion of $x$ by $t$ and $c$ in $s$ and the $g$ such that, for some $v$, the $m^{\text {th }}$ existential generalization of $s$ in $\left.t=\left\langle\vee_{s} v g\right\rangle\right\}$ for any $\left.f\right)$ ) for the $v$ such that (for some $g$, the $m^{\text {th }}$ existential generalization of $s$ in $t=\left\langle\mathrm{V}_{s} v g\right\rangle$ ) in $\left\langle\right.$ the $g$ such that, for some $v$, the $m^{\text {th }}$ existential generalization of $s$ in $t=\left\langle\mathrm{V}_{s} v g\right\rangle \wedge_{s}\left\langle\mathbf{E}_{s}\right.$ the $v$ such that, for some $g$, the $m^{\text {th }}$ existential generalization of $\left.s=\left\langle\vee_{s} v g\right\rangle\right\rangle$ in $\left.s\right\rangle$ \}

(3) the $O^{\text {th }}$ expansion of $x$ by $t$ and $c$ in $s=$ the set of all $f$ such that, for some natural number $m, f$ is in the $m^{\text {th }}$ instantial expansion of $x$ by $t$ and $c$ in $s$

(4) for any positive integer $m$,

(a) if the union of the $m-1^{\text {th }}$ expansion of $x$ by $t$ and $c$ in $s$ and $\{t(m)\}$ is consistent in $s$, then the $m^{\text {th }}$ expansion of $x$ by $t$ and $c$ in $s$ $=$ the union of the $m-1^{\text {th }}$ expansion of $x$ by $t$ and $c$ in $s$ and $\{t(m)\}$ 
(b) if the union of the $m-1^{\text {th }}$ expansion of $x$ by $t$ and $c$ in $s$ and $\{t(m)\}$ is not consistent in $s$, then the $m^{\text {th }}$ expansion of $x$ by $t$ and $c$ in $s=$ the $m-1^{\text {th }}$ expansion of $x$ by $t$ and $c$ in $s$

(5) the expansion of $x$ by $t$ and $c$ in $s=$ the set of all $f$ such that, for some natural number $m, f$ is in the $m^{\text {th }}$ expansion of $x$ by $t$ and $c$ in $s$

(e) for any term of $s a$, the set of identicals of $a$ by $x, t$, and $c$ in $s=$ the set of all $b$ such that $\left\langle\mathrm{I}_{s} b a\right\rangle$ is in the expansion of $x$ by $t$ and $c$ in $s$.

Theorem 4. If $s$ is a symbol sequence, $t$ is a proof sequence in $s, c$ is an individual constant of $s, v$ is a variable of $s$, and there are no $p$ in the range of $t$ and $f$ in the range of $p$ such that $v$ occurs in $f$, then the result of properly substituting $v$ for $c$ in $t$ in $s$ is a proof sequence in $s$.

Assume the antecedent and let $u=$ the result of properly substituting $v$ for $c$ in $t$ in $s$. By definitions 40,39, and 28,u(1) consists of a show line of $s$. Assume then that $m$ is in the domain of $u$ and $m$ is greater than 1. By definitions $40,39,38,37,36$, and $28, u(m)$ is obviously obtainable from $u(m-1)$ in $s$ since $t(m)$ is obtainable from $t(m-1)$ in $s$. Hence, by definition $33, u$ is a proof sequence in $s$ and the theorem holds.

Theorem 5. If $s$ is a symbol sequence, $f$ is a theorem of $s$ by $L_{s}, c$ is an individual constant of $s, w$ is a variable of $s$, and $w$ does not occur in $f$, then $\left\langle\wedge_{s} w\right.$ the result of properly substituting $w$ for $c$ in $f$ in $\left.s\right\rangle$ is a theorem of $s$ by $L_{s}$.

Assume the antecedent. By definition $35,\langle f\rangle$ is a proof in $s$ by $L_{s}$. Hence, by definitions $34,38,37$, and 39 , there is proof in $s$ by $L_{s p}$ such that either $\langle f\rangle$ is obtainable from $p$ by a direct proof in $s$ or $\langle f\rangle$ is obtainable from $p$ by a universal proof in $s$. By theorem 2, there is a finite proof sequence in $s r$ such that the last term of $r=p$. By definition $39, \widehat{r} \ll f \gg$ is a proof sequence in $s$. Also, there is a variable of $s v$ such that there are no $q$ in the range of $r \ll f \gg$ and $g$ in the range of $q$ such that $v$ occurs in $g$. Hence, by theorem 4 , the result of properly substituting $v$ for $c$ in $\widetilde{r}\langle f\rangle$ in $s$ is a proof sequence in $s$ and so, by definitions $40,39,38,37$, 34 , and 35 , the result of properly substituting $v$ for $c$ in $f$ in $s$ is a theorem of $s$ by $\mathrm{L}_{s}$. Hence, $\wedge v$ the result of properly substituting $v$ for $c$ in $f$ in $s$ is a theorem of $s$ by $L_{s}$. Now, $\wedge v g \rightarrow \wedge w$ the result of properly substituting $w$ for $v$ in $g$ in $s$ is a theorem of $s$ by $L_{s}$ for any variables of $s v$ and $w$ and formula of $s g$ in which $w$ is not free. Also, the result of properly substituting $w$ for $c$ in $f$ in $s=$ the result of properly substituting $w$ for $v$ in (the result of properly substituting $v$ for $c$ in $f$ in $s$ ) in $s$ since neither $v$ nor $w$ occurs in $f$. Hence, the theorem holds.

In theorems 6 through 16 and in definitions 41 through 43, it is taken for granted that $s$ is a symbol sequence, $x$ is included in the set of all formulas of $s, t$ is an enumerator of the formulas of $s, c$ is a denumerably infinite sequence, the range of $c$ is included in the set of all individual constants of $s d$ such that $d$ is not free in $f$ for any $f$ in $x$, and $c(i) \neq c(j)$ if $i \neq j$ for any positive integers $i$ and $j$. 
Theorem 6. $x$ is consistent in $s$ just in case the expansion of $x$ by $t$ and $c$ in $s$ is consistent in $s$.

Assume first that $x$ is consistent in $s$. The expansion of $x$ by $t$ and $c$ in $s$ is obviously consistent in $s$ if, for any natural number $n$, the $n^{\text {th }}$ instantial expansion of $x$ by $t$ and $c$ in $s$ is consistent in $s$. Since $x$ is consistent in $s$ and the $0^{\text {th }}$ instantial expansion of $x$ by $t$ and $c$ in $s=x$, it is sufficient to show that, for any natural number $n$, if the $n+1^{\text {th }}$ instantial expansion of $x$ by $t$ and $c$ in $s$ is not consistent in $s$, then the $n^{\text {th }}$ instantial expansion of $x$ by $t$ and $c$ in $s$ is not consistent in $s$. Assume then that $n$ is a natural number and that the $n+1^{\text {th }}$ instantial expansion of $x$ by $t$ and $c$ in $s$ is not consistent in $s$. Also, let $e=$ the $n^{\text {th }}$ instantial expansion of $x$ by $t$ and $c$ in $s$. By definition 40, we then know that, for some variable of $s v$, formula of $s f$, and individual constant of $s d$ which is free neither in $f$ nor in some $g$ in $e$, e implies $N<\mathrm{V} v f \rightarrow$ the result of properly substituting $d$ for $v$ in $f \wedge \mathrm{E} v$ in $s>$ in $s$. Hence, for some finite sequence $u$ whose range is included in $e$, both the conjunction for $u$ of $s \rightarrow \vee v f$ and the conjunction for $u$ of $s \rightarrow N$ the result of properly substituting $d$ for $v$ in $f \wedge E v$ in $s$ are theorems of $s$ by $L_{s}$. Let $w$ be a variable of $s$ which occurs neither in the conjunction for $u$ of $s$ nor in $\vee v f$. By theorem $5, \wedge w$ the result of properly substituting $w$ for $d$ in <the conjunction for $u$ of $s \rightarrow N$ the result of properly substituting $d$ for $v$ in $f \wedge \mathbf{E} v$ in $s>$ in $s$ is a theorem of $s$ by $\mathrm{L}_{s}$. Since $\wedge w\langle c \rightarrow h\rangle$ $\rightarrow\langle c \rightarrow \wedge w h\rangle$ is a theorem of $s$ by $L_{s}$ for any variable of $s w$ and formulas of $s c$ and $h$ such that $w$ is not free in $c$, since $d$ is not free in the conjunction for $u$ of $s$, and since $v$ is free in $\mathrm{E} v$, it follows that the conjunction for $u$ of $s \rightarrow \wedge w N<$ the result of properly substituting $w$ for $d$ in the result of properly substituting $d$ for $v$ in $f$ in $s$ in $s \wedge \mathrm{E} w>$ is a theorem of $s$ by $\mathrm{L}_{s}$. Now, both $\wedge w N\langle r \wedge \mathrm{E} w>\rightarrow N \vee w r$ and $\vee v f \rightarrow \vee w$ the result of properly substituting $w$ for $v$ in $f$ in $s$ are theorems of $s$ by $L_{s}$ for any formula of $s r$. Hence, the conjunction for $u$ of $s \rightarrow N \vee w$ the result of properly substituting $w$ for $d$ in the result of substituting $d$ for $v$ in $f$ in $s$ in $s \wedge \vee w$ the result of properly substituting $w$ for $v$ in $f$ in $s$ is a theorem of $s$ by $L_{s}$. Assume that $v$ is not free in $f$. Then, since $d$ is not free in $f$, the result of properly substituting $w$ for $d$ in the result of properly substituting $d$ for $v$ in $f$ in $s$ in $s$ = the result of properly substituting $w$ for $v$ in $f$ in $s=f$ and so the conjunction for $u$ of $s \rightarrow N \vee w f \wedge \vee w f$ is a theorem of $s$ by $\mathrm{L}_{s}$; that is, $e$ is not consistent in $s$. Assume on the other hand that $v$ is free in $f$. Then, since $w$ does not occur in $f$, the result of properly substituting $w$ for $d$ in the result of properly substituting $d$ for $v$ in $f$ in $s$ in $s=$ the result of properly substituting $w$ for $v$ in $f$ in $s$ and so $e$ is again not consistent in $s$. Hence, the expansion of $x$ by $t$ and $c$ in $s$ is consistent in $s$. On the other hand, $x$ is obviously consistent in $s$ if the expansion of $x$ by $t$ and $c$ in $s$ is since $x$ is included in this expansion. Hence, the theorem holds.

In theorems 7 through 16 , it is taken for granted that $x$ is consistent in $s$.

Theorem 7. If $f$ is a formula of $s$, then

(1) the expansion of $x$ by $t$ and $c$ in $s$ implies $f$ in $s$ just in case $f$ is a member of the expansion of $x$ by $t$ and $c$ in $s$ 
(2) if the expansion of $x$ by $t$ and $c$ in $s$ implies $f$ in $s$, then the expansion of $x$ by $t$ and $c$ in $s$ does not imply $\left\langle N_{s} f\right\rangle$ in $s$

(3) if the expansion of $x$ by $t$ and $c$ in $s$ does not imply $f$ in $s$, then the expansion of $x$ by $t$ and $c$ in $s$ implies $\left\langle N_{s} f\right\rangle$ in $s$.

Assume that $f$ is a formula of $s$. (1) follows immediately from theorem 6 , definition 40 , and the fact $f \rightarrow f$ is a theorem of $s$ and (2) follows immediately from theorem 6 and definition 40 . To show (3), assume its antecedent and let $e=$ the expansion of $x$ by $t$ and $c$ in $s$. By definition 40, the union of $e$ and $\{f\}$ is consistent in $s$ only if $f$ is in $e$. Since $f$ cannot be in $e$, it follows that $e$ implies $f \rightarrow N f$ and so $N f$ in $s$. Hence, (3) holds and so the theorem holds.

Theorem 8. If $a$ and $b$ are terms of $s$, then

(1) either the set of identicals of $a$ by $x, t$, and $c$ in $s$ is not empty or the set of identicals of $b$ by $x, t$, and $c$ in $s$ is not empty just in case (the set of identicals of $a$ by $x, t$, and $c$ in $s=$ the set of identicals of $b$ by $x$, $t$, and $c$ in $s$ just in case $\left\langle\mathbf{I}_{s} a b\right\rangle$ is in the expansion of $x$ by $t$ and $c$ in $s)$

(2) the set of identicals of $a$ by $x, t$, and $c$ in $s$ is empty and the set of identicals of $b$ by $x, t$, and $c$ in $s$ is empty just in case $\left\langle N_{s}\left\langle E_{s} a\right\rangle\right.$ is in the expansion of $x$ by $t$ and $c$ in $s$ and $\left\langle N_{s}\left\langle\mathbf{E}_{s} b\right\rangle\right\rangle$ is in the expansion of $x$ by $t$ and $c$ in $s$.

Assume the antecedent. To show (1), assume first the left side of (1) and the left side of the right side of (1). It follows that, for some term of $s d, d \mathbf{I} a$ and $d \mathbf{I} b$ are in the expansion of $x$ by $t$ and $c$ in $s$. Since $d \mathbf{I} a \wedge d \mathbf{I} b \rightarrow a \mathbf{I} b$ is a theorem of $s$ by $\mathrm{L}_{s}$, we then have the right side of the right side of (1) by theorem 7. Assume instead the right side of the right side of (1). It follows that, for any term of $s d, d \mathbf{I} a$ is in the expansion of $x$ by $t$ and $c$ in $s$ just in case $d \mathbf{I} b$ is since $a \mathbf{I} b \rightarrow\langle d \mathbf{I} a \leftrightarrow d \mathbf{I} b\rangle$ is a theorem $s$ by $L_{s}$. Hence, we have the left side of the right side of (1). Assume now the right side of (1). If the sets of identicals of both $a$ and $b$ by $x, t$ and $c$ in $s$ are empty, then they are identical and so not empty by the right side of (1). Hence, one of them is not empty and so (1) holds.

To show (2), assume its left side. Since $a \mathbf{I} a$ and $b \mathbf{I} b$ are then not in the expansion of $x$ by $t$ and $c$ in $s$ and $N a \mathrm{I} a \rightarrow N \mathrm{E} a$ is a theorem of $s$ by $\mathrm{L}_{s}$ for any term of $s a$, the right side of (2) holds by theorem 7. Assume finally that the right side of (2) holds. Since $N E a \rightarrow N d \mathbf{I} a$ is a theorem of $s$ by $\mathrm{L}_{s}$ for any terms of $s a$ and $d$, it follows by theorem 7 that neither $d \mathbf{I} a$ nor $d \mathbf{I} b$ is in the expansion of $x$ by $t$ and $c$ in $s$ for any term of $s d$. Hence, (2) holds and so the theorem holds.

Theorem 9. If $a$ is a term of $s$ and the set of identicals of $a$ by $x, t$ and $c$ in $s$ is not empty, then, for some individual constant of $s b$, the set of identicals of $b$ by $x, t$, and $c$ in $s=$ the set of identicals of $a$ by $x, t$, and $c$ in $s$.

Assume the antecedent. It follows that, for some term of $s d, d \mathbf{I} a$ is in the expansion of $x$ by $t$ and $c$ in $s$. Since $d \mathbf{I} a \rightarrow \vee v v \mathbf{I} a$ is a theorem of $s$ by $L_{s}$ for any variable of $s$ not free in $a v$, it follows by definitions 40 and 28 and theorem 7 that, for some individual constant of $s b$, both $\mathbf{E} b$ and $b \mathbf{I} a$ are 
in the expansion of $x$ by $f$ and $c$ in $s$. Hence, by theorem 8, we have the consequent.

Theorem 10. If $v$ is a variable of $s$ and $f$ is a formula of $s$, then $\left\langle\wedge_{s} v f\right\rangle$ is in the expansion of $x$ by $t$ and $c$ in $s$ just in case, for any individual constant of $s b$, if the set of identicals of $b$ by $x, t$, and $c$ in $s$ is not empty, then the result of properly substituting $b$ for $v$ in $f$ in $s$ is in the expansion of $x$ by $t$ and $c$ in $s$.

Assume the antecedent. If the left side of the consequent holds, $b$ is an individual constant of $s$, and the set of identicals of $b$ by $x, t$, and $c$ in $s$ is not empty, then $\mathrm{E} b$ is in the expansion of $x$ by $t$ and $c$ in $s$. Hence, since $\wedge v f \rightarrow\langle\mathbf{E} b \rightarrow$ the result of properly substituting $b$ for $v$ in $f$ in $s\rangle$ is a theorem of $s$ by $\mathrm{L}_{s}$, the result of properly substituting $b$ for $v$ in $f$ in $s$ is in the expansion of $x$ by $t$ and $c$ in $s$ by theorem 7. Assume then that the right side of the consequent holds. If $\wedge v f$ is not in the expansion of $x$ by $t$ and $c$ in $s$, then $\vee v N f$ is by theorem 7 since $\wedge \wedge v f \rightarrow \vee v N f$ is a theorem of $s$ by $L_{s}$. But, by definition 40, theorem 7, and definition 28, if $\vee v N f$ is in the expansion of $x$ by $t$ and $c$ in $s$, then, for some individual constant of $s b$, the set of identicals of $b$ by $x, t$, and $c$ in $s$ is not empty and $N$ the result of properly substituting $b$ for $v$ in $f$ in $s$ is in the expansion of $x$ by $t$ and $c$ in $s$. Also, by theorem 7, there is no formula of $s g$ such that both $g$ and $N g$ are in the expansion of $x$ by $t$ and $c$ in $s$. Hence, $\wedge v f$ is in the expansion of $x$ by $t$ and $c$ in $s$ and the theorem holds.

Definition 41. $a$ is an assigner with respect to $x, t, c$, and $s$ just in case, for any term or formula of $s u, a$ is an assigner for $u$ in (the set of all $y$ such that $y$ is not empty and, for some term of $s b, y=$ the set of identicals of $b$ by $x, t$ and $c$ in $s$ ) with respect to $s$.

Definition 42. If $x$ is consistent in $s$, then

(1) if $b$ is a term of $s$ and the set of identicals of $b$ by $x, t$, and $c$ in $s$ is not empty, then the $1^{\text {th }}$ identical of $b$ with respect to $x, t$, and $c$ in $s=$ the $d$ such that, for some positive integer $n, d=$ the $n^{\text {th }}$ individual constant of $s$, $d$ is in the set of identicals of $b$ by $x, t$, and $c$ in $s$, and there is no positive integer $m$ smaller than $n$ such that the $m^{\text {th }}$ individual constant of $s$ is in the set of identicals of $b$ by $x, t$, and $c$ in $s$

(2) if both either $u$ is a term of $s$ or $u$ is a formula of $s$ and $a$ is an assigner with respect to $x, t, c$, and $s$, then the sequence of individual constants of $s$ for $u$ with respect to $a, x, t$, and $c=$ the $y$ satisfying the following conditions:

(a) $y$ is a function

(b) the domain of $y=$ the domain of the sequence of variables of $s$ free in $u$

(c) for any $j$ in the domain of $y$, either $a$ (the $j^{\text {th }}$ variable of $s$ free in $u$ ) is empty and $y(j)=$ the $1^{\text {th }}$ individual constant of $s$ or, for some term of $s b, a$ (the $j^{\text {th }}$ variable of $s$ free in $u$ ) $=$ the set of identicals of $b$ by $x, t$, and $c$ in $s\}$ and $y(j)=$ the $1^{\text {th }}$ identical of $b$ with respect to $x, t$, and $c$ in $s$.

Definition 43. Fxtcs $=$ the $i$ satisfying the following conditions:

(1) $i$ is a function

(2) the domain of $i=$ the set of all descriptive constants of $s$ 
(3) for any individual constant of $s d$, either the set of identicals of $d$ by $x, t$, and $c$ in $s$ is empty and $i(d)=$ the empty set or the set of identicals of $d$ by $x, t$, and $c$ in $s$ is not empty and $i(d)=\{$ the set of identicals of $d$ by $x, t$, and $c$ in $s$ \}

(4) for any positive integer $m$ and any $b$,

(a) if $b$ is an $m$-place operation symbol of $s$, then $i(b)=$ the set of all ordered pairs $v, w$ such that, for some $u$,

(1) $v$ and $u$ are $m$-term sequences

(2) the range of $u$ is included in the set of all terms of $s a$ such that the set of identicals of $a$ by $x, t$, and $c$ in $s$ is not empty

(3) for any $j$ in the domain of $v, v(j)=$ the set of identicals of $u(j)$ by $x, t$, and $c$ in $s$

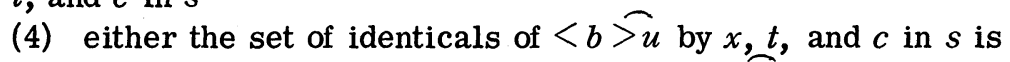
empty and $w=$ the empty set or the set of identicals of $\langle b\rangle u$ by $x, t$, and $c$ in $s$ is not empty and $w=\{$ the set of identicals of $\langle b>u$ by $x, t$, and $c$ in $s\}$

(b) if $b$ is an $m$-place predicate of $s$, then $i(b)=$ the set of all $m$-term sequences $v$ such that, for some $m$-term sequence $u$ whose range is included in the set of all terms of $s$,

(1) $\langle b>u$ is in the expansion of $x$ by $t$ and $c$ in $s$

(2) for any $j$ in the domain of $v, v(j)=$ the set of identicals of $u(j)$ by $x, t$, and $c$ in $s$

(5) for any natural numbers $l$ and $m$, if either $l$ or $m$ is a positive integer, then for any $b$,

(a) if $b$ is a $O$-place $l$-term $m$-formula term making variable binder of $s$, then

(1) $i(b)$ is a function

(2) the domain of $i(b)=$ the set of all 2-term sequences $u$ such that $u(1)$ is an $l$-term sequence whose range is included in the set of all terms of $s$ and $u(2)$ is an $m$-term sequence whose range is included in the set of all formulas of $s$

(3) for any $u$ in the domain of $i(b)$, either the set of identicals of $(\langle b>\vec{u}(1)) \hat{u}(2)$ by $x, t$, and $c$ in $s$ is empty and $(i(b))(u)=$ the empty set or the set of identicals of $(\langle b>u(1) \hat{u}(2)$ by $x, t$, and $c$ in $s$ is not empty and $(i(b))(u)=\{$ the set of identicals of $(\langle b>u(1)) u(2)$ by $x, t$, and $c$ in $s\}$

(b) if $b$ is a 0 -place $l$-term $m$-formula formula making variable binder of $s$, then $i(b)=$ the set of all 2 -term sequences $u$ such that $(<b \widehat{>u}(1) \hat{)}(2)$ is in the expansion of $x$ by $t$ and $c$ in $s$.

In theorems 11 through 16 , it is taken for granted that $i=\mathrm{F} x$ tcs and that $j=$ the set of all $y$ such that $y$ is not empty and, for some term of $s a$, $y=$ the set of identicals of $a$ by $x, t$, and $c$ in $s$.

Theorem 11. If $m$ is a positive integer and $b$ is an $m$-place operation symbol of $s$, then $i(b)$ is a function.

Assume the antecedent and that the ordered pairs $p, q$ and $p, r$ are in $i(b)$. We must show that $q=r$. From the assumption and definition 43 , we know that, for some $u$ and $v, u, v$, and $p$ are $m$-term sequences, the ranges of both $u$ and $v$ are included in the set of all terms of $s a$ such the set of identicals of $a$ by $x, t$, and $c$ in $s$ is not empty, $p(l)=$ the set of identicals of 
$u(l)$ by $x, t$, and $c$ in $s=$ the set of identicals of $v(l)$ by $x, t$, and $c$ in $s$ for any $l$ in the domain of $p$, either the set of identicals of $\langle b\rangle u$ by $x, t$, and $c$ in $s$ is empty and $q$ is empty or the set of identicals of $\langle b>u$ by $x, t$, and $c$ in $s$ is not empty and $q=$ \{the set of identicals of $\langle b\rangle u$ by $x, t$, and $c$ in $s\}$, and either the set of identicals of $\langle b\rangle \bar{v}$ by $x, t$, and $c$ in $s$ is empty and $r$ is empty or the set of identicals of $\langle b\rangle_{v}$ by $x, t$, and $c$ in $s$ is not empty and $r=\{$ the set of identicals of $\langle b\rangle v$ by $x, t$, and $c$ in $s\}$. From the second and third of these consequences, it follows by theorem 8 that $u(l) \mathrm{I} v(l)$ is in the expansion of $x$ by $t$ and $c$ in $s$ for any $l$ in the domain of $u$. Let $w=$ the $m$-term sequence $w$ such that, for any $l$ in the domain of $w, w(l)$ $=u(l) \mathbf{I} v(l)$ and let $h=$ the conjunction for $w$ of $s$. If the sets of identicals of both $\langle b\rangle u$ and $\langle b\rangle v$ by $x, t$, and $c$ in $s$ are empty, then both $q$ and $r$ are empty and so $q=r$. Assume then that the set of identicals of $<b>\widehat{u}$ by $x, t$, and $c$ in $s$ is not empty. Since both $h \wedge a \mathbf{I}<b>u \rightarrow a \mathbf{I}<b>v$ and $a \mathrm{I}\left\langle b>u \wedge a \mathbf{I}<b>\widehat{v} \rightarrow\left\langle b>u \mathbf{I}<b>\widehat{v}\right.\right.$ are theorems of $s$ by $\mathrm{L}_{s}$ for any term of $s a$, it follows by theorem 7 that $\langle b>u \mathrm{I}\langle b\rangle v$ is in the expansion of $x$ by $t$ and $c$ in $s$. Since the set of identicals of $\langle b>v$ by $x, t$, and $c$ in $s$ is then not empty, $q=r$ by theorem 8. By the same reasoning, $q=r$ if the set of identicals of $\langle b\rangle v$ by $x, t$, and $c$ in $s$ is not empty. Hence, the theorem holds.

Theorem 12. If $m$ is a positive integer and $b$ is an $m$-place predicate of $s$, then $i(b)$ is included in the set of all $m$-term sequences whose ranges are included in $j$.

Assume the antecedent and that $v$ is in $i(b)$. By definition $43, v$ is an $m$-term sequence and, for some $m$-term sequence $u$ whose range is included in the set of all terms of $s,\langle b\rangle u$ is in the expansion of $x$ by $t$ and $c$ in $s$ and, for any $l$ in the domain of $v, v(l)=$ the set of identicals of $u(l)$ by $x$, $t$, and $c$ in $s$. Since $\left\langle b>u \rightarrow u(l) \mathrm{I} u(l)\right.$ is a theorem of $s$ by $L_{s}$ for any $l$ in the domain of $u$, it follows by theorem 7 that the set of identicals of $u(l)$ by $x, t$, and $c$ in $s$ is not empty for any $l$ in the domain of $u$. Hence, the range of $v$ is included in $j$.

Theorem 13. $i\left(E_{s}\right)=$ the set of all 1 -term sequences whose ranges are included in $j$ and $i\left(\mathbf{I}_{s}\right)=$ the set of all 2-term sequences $v$ such that, for some $m$ in $j$, the range of $v=\{m\}$.

By definition 43, $i(E)=$ the set of all 1-term sequences $v$ such that, for some term of $s a, \mathrm{E} a$ is in the expansion of $x$ by $t$ and $c$ in $s$ and $v=<$ the set of identicals of $a$ by $x, t$ and $c$ in $s>$. Also, $i(\mathbf{I})=$ the set of all 2 -term sequences $v$ such that, for some terms of $s a$ and $b, a \mathbf{I} b$ is in the expansion of $x$ by $t$ and $c$ in $s$ and $v=<$ the set of identicals of $a$ by $x, t$, and $c$ in $s$ the set of identicals of $b$ by $x, t$, and $c$ in $s>$. Hence, the theorem holds.

Theorem 14. $i$ is an interpreter with respect to $s$.

This follows from definitions 21 and 43 and theorems 11 through 13 .

Theorem 15. If $v$ is a variable of $s, f$ is a formula of $s$, and $a$ is an assigner for $\left\langle\Lambda_{s}\right.$ vf $\rangle$ in $j$ with respect to $s$, then $\left\langle\Lambda_{s} v f\right\rangle$ satisfies $i$ and $a$ just in case, for any individual constant of $s d$, if the set of identicals 
of $d$ by $x, t$, and $c$ in $s$ is not empty, then $f$ satisfies $i$ and $a(\{$ the set of identicals of $d$ by $x, t$, and $c$ in $s\}$.$) .$

The theorem follows immediately from theorem 14, definition 22 , definition 43, and theorem 9 .

Theorem 16. If $f$ is a formula of $s$, then, for any $a$, if $a$ is an assigner with respect to $x, t, c$, and $s$, then $f$ satisfies $i$ and $a$ just in case the result of properly substituting (the sequence of individual constants of $s$ for $f$ with respect to $a, x, t$, and $c$ ) for (the sequence of variables of $s$ free in $f$ ) in $f$ in $s$ is in the expansion of $x$ by $t$ and $c$ in $s$.

For any term of $s u$, let $\mathbf{I} u=$ the set of identicals of $u$ by $x, t$, and $c$ in $s$. Also, for any term or formula of $s u$, let $\mathbf{V} u=$ the sequence of variables of $s$ free in $u$ and, for any assigner with respect to $x, t, c$, and $s a$, let C $a u$ $=$ the sequence of individual constants of $s$ for $u$ with respect to $a, x, t$, and let $\mathrm{I} a u=$ the interpretation of $u$ with respect to $i$ and $a$. Also, for any $u$ and $v$, if either $u$ and $v$ are terms of $s$ or, for some natural number $z, u$ and $v$ are $z$-term sequences whose ranges are included in the set of all terms of $s$, then, for any term or formula of $s w$, let $\mathbf{S} v u w=$ the result of properly substituting $v$ for $u$ in $w$ in $s$. Finally, for any natural number $z$, let $\mathbf{T} z=$ the set of all $z$-level terms of $s u$ such that, for any assigner with respect to $x, t, c$, and $s a, \mathbf{I} a u=\mathbf{I} a \mathbf{S}(\mathbf{C} a u)(\mathbf{V} u) u$ and either $\mathbf{I S}(\mathbf{C} a u)(\mathbf{V} u) u$ is empty and $\mathbf{I} a \mathbf{S}(\mathbf{C} a u)(\mathbf{V} u) u$ is empty or not and $\mathbf{I} a \mathbf{S}(\mathbf{C} a u)(\mathbf{V} u) u=\{\mathbf{I S}(\mathbf{C} a u)(\mathbf{V} u) u$ and let $\mathbf{F} z=$ the set of all $z$-level formulas of $s f$ such that, for any assigner with respect to $x, t, c$, and $s a$, both $f$ satisfies $i$ and $a$ just in case $\mathbf{S}(\mathbf{C} a f)(\mathbf{V} f) f$ satisfies $i$ and $a$ and $\mathbf{S}(\mathbf{C} a f)(\mathbf{V} f) f$ satisfies $i$ and $a$ just in case $\mathbf{S}(\mathbf{C} a f)(\mathbf{V} f) f$ is in the expansion of $x$ by $t$ and $c$ in $s$.

To establish the theorem, it is sufficient to show that, for any natural numbers $z$, every $z$-level formula of $s$ is in $F z$. Hence, it is sufficient to show that

(1) every 0 -level term of $s$ is in To

(2) for any natural number $z$,

(a) if every $z$-level term of $s$ is in T $z$, then every $z$-level formula of $s$ is in $\mathbf{F} z$

(b) if every $z$-level formula of $s$ is in F $z$, then every $z+1$-level term of $s$ is in $\mathrm{T} z+1$.

All variables and individual constants of $s$ are obviously in $\mathrm{T} z$ for any natural number $z$ and so (1) holds. To show (2), assume that $z$ is a natural number. To show (a) of (2), assume that every $z$-level term of $s$ is in T $z$. Assume first that $m$ is a positive integer, $b$ is an $m$-place predicate of $s, u$ is an $m$-term sequence whose range is included in the set of all $z$-level terms of $s$, and $a$ is an assigner with respect to $x, t, c$, and $s$. Let $v=$ the $m$-term sequence $v$ such that, for any $l$ in the domain of $v, v(l)=\mathbf{S}(\mathbf{C} a u(l))$ $(\mathrm{V} u(l)) u(l)$. If, for some $l$ in the domain of $u$, I $a u(l)$ is empty, then, by the inductive assumption, both $\operatorname{I} a v(l)$ and $\mathbf{I} v(l)$ are empty and so $\langle b\rangle v$ is not in the expansion of $x$ by $t$ and $c$ in $s$. Since both $\langle b>u$ and $\langle b>v$ then do not satisfy $i$ and $a$ by definition 22 and $\langle b>v=\mathbf{S}(\mathbf{C} a<b>u)(\mathbf{V}<b>u)<b>u$, $\langle b\rangle \hat{u}$ is then in $\mathbf{F} z$. Assume on the other hand that, for every $l$ in the do- 
main of $u, \mathbf{I} a u(l)$ is not empty. By the inductive assumption, $\mathbf{I} a u(l)=\mathbf{I} a v(l)=$ $\{\mathbf{I} v(l)\}$ and $\mathbf{I} v(l)$ is not empty for any $l$ in the domain of $u$. Let $w=$ the $m$-term sequence $w$ such that, for any $l$ in the domain of $w, w(l)=\mathbf{I} v(l)$. By definitions 43 and 22, each of $\langle b \widehat{\rangle u}$ and $\langle b \widehat{\nabla v}$ satisfies $i$ and $a$ just in case there is an $m$-term sequence $y$ whose range is included in the set of all terms of $s$ such that $\langle b\rangle y$ is in the expansion of $x$ by $t$ and $c$ in $s$ and, for any $l$ in the domain of $w, w(l)=\mathrm{I} y(l)$; that is, by theorems 8 and 7 , just in case $\langle b>v$ is in the expansion of $x$ by $t$ and $c$ in $s$. Since $\langle b>v=$ $\mathbf{S}(\mathbf{C} a<b>u)(\mathbf{V}<b>\widehat{>})<b>\widehat{u},<b>u$ is then again in $\mathbf{F} z$.

By theorem 7, $N f$ and $f b g$ are in $\mathbf{F} z$ if $f$ and $g$ are and $b$ is a standard binary connective of $s$. Also, if Llmbu (the set of all $z$-level terms of $s) f(\mathbf{F} z) s$ and $g=(\langle b>u) f$, then, since no variable of $s$ is free in $g$, $g=\mathbf{S}(\mathbf{C} a g)(\mathbf{V} g) g$ for any assigner with respect to $x, t, c$, and $s a$ and so is in $\mathbf{F} z$ by definitions 22 and 43. Assume then that $v$ is a variable of $s$ and that $f$ is in $\mathbf{F} z$. If either $j$ is empty or $v$ is not free in $f$, then $\wedge v f$ is obviously in $\mathbf{F} z$. Assume instead that $j$ is not empty, that $v$ is free in $f$, and that $a$ is an assigner with respect to $x, t, c$, and $s$. By definitions 42 and $41, \mathrm{I} b$ is not empty for any $b$ in the range of $C a \wedge v f$. By theorem 15, $\wedge v f$ satisfies $i$ and $a$ just in case, for any individual constant of $s b$, if $\mathbf{I} b$ is not empty, then $f$ satisfies $i$ and $a\left(\begin{array}{l}v \\ \{\mathfrak{I} b\}\end{array}\right)$. Since, for any individual constant of $s b$ such that $\mathbf{I} b$ is not empty, $a\left(\begin{array}{l}v \\ \{\mathfrak{I} b\}\end{array}\right)$ is an assigner with respect to $x, t, c$, and $s$, it follows by the inductive assumption that $\wedge v f$ satisfies $i$ and $a$ both just in case $\mathbf{S}\left(\mathbf{C} a\left({ }_{\{\mathrm{I} b\}}^{v}\right) f\right)(\mathbf{V} f) f$ satisfies $i$ and $a\left({ }_{\{\mathrm{I} b\}}^{v}\right)$ and just in case $\mathbf{S}\left(\mathbf{C} a\left(\begin{array}{l}v \\ \{\mathbf{I} b\}\end{array}\right) f\right)(\mathbf{V} f) f$ is in the expansion of $x$ by $t$ and $c$ in $s$ for any individual constant of $s b$ such that $I b$ is not empty. Now, by definition 42, $\mathbf{S}\left(\mathbf{C} a\left(\begin{array}{l}v \\ \{\mathbf{I} b\}\end{array}\right) f\right)(\mathbf{V} f) f=\mathbf{S}$ (the $1^{\text {th }}$ identical of $b$ with respect to $x, t$, and $c$ in $\left.s\right) v$

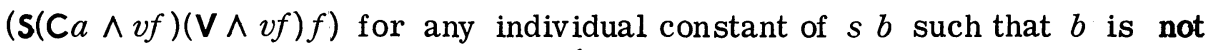
empty. Also, for any such $b$, (the $1^{\text {th }}$ identical of $b$ with respect to $x, t$, and $c$ in $s$ ) $\mathbf{I} b$ is in the expansion of $x$ by $t$ and $c$ in $s$. It follows that $\mathbf{S}$ (the $1^{\text {th }}$ identical of $b$ with respect to $x, t$ and $c$ in $s) v(\mathbf{S}(\mathbf{C} a \wedge v f)(\mathbf{V} \wedge v f) f) \leftrightarrow$ $\mathbf{S} b v(\mathbf{S}(\mathbf{C} a \wedge v f)(\mathbf{V} \wedge v f) f)$ both satisfies $i$ and $a\left(\begin{array}{l}v \\ \{\mid \boldsymbol{b} b\}\end{array}\right)$ and is in the expansion of $x$ by $t$ and $c$ in $s$ for any $\operatorname{such} b$. Hence, $\wedge v f$ satisfies $i$ and $a$ both just in case $\mathbf{S} b v(\mathbf{S}(\mathbf{C} a \wedge v f)(\mathbf{V} \wedge v f) f)$ satisfies $i$ and $a\left(\begin{array}{c}v \\ \{\mathrm{I} b\}\end{array}\right)$ and just in case

$\mathbf{S} b v(\mathbf{S}(\mathbf{C} a \wedge v f)(\mathbf{V} \wedge v f) f)$ is in the expansion of $x$ by $t$ and $c$ in $s$ for any individual constant of $s b$ such that $\mathrm{I} b$ is not empty; that is, by theorems 15 and 10, both just in case $\wedge v \mathbf{S}(\mathbf{C} a \wedge v f)(\vee \wedge v f) f$ satisfies $i$ and $a$ and just in case $\wedge v \mathbf{S}(\mathbf{C} a \wedge v f)(\vee \wedge v f) f$ is in the expansion of $x$ by $t$ and $c$ in $s$. Since $\mathbf{S}(\mathbf{C} a \wedge v f)(\mathbf{V} \wedge v f) \wedge v f=\wedge v \mathbf{S}(\mathbf{C} a \wedge v f)(\mathbf{V} \wedge v f) f$, it follows that $\wedge v f$ is in $\mathbf{F} z$.

From the fact that, for any variable of $s v$ and $f$ in $\mathbf{F} z$, both $N f$ and $\wedge v f$ are in $\mathbf{F} z$, it follows immediately that $\vee v f$ is in $\mathbf{F} z$ for $\operatorname{such} v$ and $f$. Hence, (a) of (2) holds.

To show (b) of (2), assume that every $z$-level formula of $s$ is in F $z$. 
We already know that all variables and individual constants of $s$ are in $\mathbf{T} z+1$. Assume then that $m$ is a positive integer, $b$ is an $m$-place operation symbol of $s, u$ is an $m$-term sequence whose range is included in $\mathrm{T} z+1$, and $a$ is an assigner with respect to $x, t, c$, and $s$. Let $v=$ the $m$-term sequence $v$ such that, for any $l$ in the domain of $v, v(l)=$ $\mathbf{S}\left(\mathbf{C}_{a} u(l)\right)(\mathbf{V} u(l)) u(l)$. If, for some $l$ in the domain of $u, \mathbf{I} a u(l)$ is empty, then, by the inductive assumption, both $\mathbf{I} a v(l)$ and $\mathbf{I} v(l)$ are empty and so $N \mathrm{E}<b>v$ is in the expansion of $x$ by $t$ and $c$ in $s$. Since both $\mathrm{I} a<b>u$ and $\mathbf{I} a<b>v$ are empty by definition 22 and $\langle b \widehat{>v}=\mathrm{S}(\mathrm{C} a<b>\mathrm{u})(\mathbf{V}<b>\widehat{\sim})$ $\langle b>u,\langle b>u$ is then in $T z+1$. Assume on the other hand that, for every $l$ in the domain of $u, \mathbb{I} a u(1)$ is not empty. By the inductive assumption, $\operatorname{la} u(l)=\mathbf{I} a v(l)=\{\mathbf{I} v(l)\}$ and $\mathbf{I} v(l)$ is not empty for any $l$ in the domain of $u$. Let $w=$ the $m$-term sequence $w$ such that, for any $l$ in the domain of $w$, $w(l)=\mathbf{I} v(l)$. Since $\mathbf{I} a<b>\widehat{\sim} u=(i(b))(w)=\mathbf{I} a<b>v$, since $(i(b))(w)$ is empty if $\mathbf{I}<b>v$ is and is $\{\mathbf{I}<b>v\}$ if $\mathbf{I}<b>v$ is not empty by definition 43, and since $\langle b>v=S(C a<b>u)(V<b>u)<b>u,\langle b>u$ is again in $\mathrm{T} z+1$.

Assume now that $\operatorname{Klmbu}(\mathrm{T} z+1) f$ (the set of all $z$-level formulas of $s) s$ and let $g=(\langle b\rangle u \hat{f}$. Since no variable of $s$ is free in $g, g=$ $\mathbf{S}(\mathrm{C} a g)(\mathrm{V} g) g$ for any assigner with respect to $x, t, c$, and $s a$. Then, since, by definitions 22 and 43, either $1 \mathrm{~g}$ is empty and $\mathrm{lag}$ is empty or not and $\mathbf{I} a g=\{1 g\}$ for any assigner with respect to $x, t, c$, and $s a, g$ is in $\mathrm{T} z+1$. Assume finally that $v$ is a variable of $s, f$ is a $z$-level formula of $s$, and $a$ is an assigner with respect to $x, t, c$, and $s$. Let $w$ be a variable of $s$ such that $w \neq v$ and $w$ does not occur in $f$. Obviously, (there is an $l$ in $j$ such that, for any $m$ in $j, f$ satisfies $i$ and $a\left(\begin{array}{c}v \\ \{m\}\end{array}\right)$ just in case $\left.l=m\right)$ just in case $\vee w \wedge v<f \leftrightarrow v \mathbf{I} w>$ satisfies $i$ and $a$. Let $g=\mathbf{S}(\mathbf{C} a \vee w \wedge v<f \leftrightarrow v \mathbf{I} w>)$ $(\mathbf{V} \vee w \wedge v<f \leftrightarrow v \mathbf{I} w>) \vee w \wedge v<f \leftrightarrow v \mathbf{I} w>=\vee w \wedge v<\mathbf{S}(\mathbf{C} a \mathbf{1} v f)(\mathbf{\vee} v f) f \leftrightarrow$ $v \mathbf{I} w\rangle$. Since $\vee w \wedge v\langle f \leftrightarrow v \mathbf{I} w\rangle$ is a $z$-level formula of $s$, it satisfies $i$ and $a$ both just in case $g$ does and just in case $g$ is in the expansion of $x$ by $t$ and $c$ in $s$. Assume now that, for some $l$ in $j$ and any $m$ in $j, f$ satisfies $i$ and $a(\underset{\{m\}}{v})$ just in case $m=l$. Hence, $g$ satisfies $i$ and $a$ and $g$ is in the expansion of $x$ by $t$ and $c$ in $s$. It follows both that, for some $k$ in $j$ and any $m$ in $j, \mathbf{S}(\mathbf{C} a \mathbf{l} v f)(\mathbf{V} \boldsymbol{v} v f) f$ satisfies $i$ and $a(\{m\})$ just in case $m=k$ and that

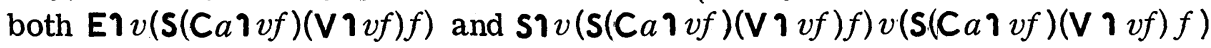
are in the expansion of $x$ by $t$ and $c$ in $s$. Assume first that $v$ is not free in $f$. Then, since $f$ satisfies $i$ and $a\left(\begin{array}{c}v \\ \{l\}\end{array}\right), f$ satisfies $i$ and $a\left(\begin{array}{l}v \\ \{m\}\end{array}\right)$ for any $m$ in $j$. Hence, $m=l$ for any $m$ in $j$ and so $k=l$ and $\operatorname{I\eta } v(\mathbf{S}(\mathbf{C} a \boldsymbol{\eta} v f)(\mathbf{V} v f) f)=l$.

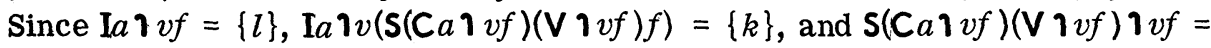
$\boldsymbol{\eta} v(\mathbf{S}(\mathbf{C} a \mathfrak{\urcorner} v f)(\mathbf{V} \boldsymbol{\urcorner} v f) f)$, it follows that $\mathbf{1} v f$ is in $\mathbf{T} z+1$. Assume then that $v$ is free in $f$. We know by theorem 9 that, for some individual constants of $s b$ and $d, b$ is in $l$ and $d \mathbf{I} \mathbf{I} v(\mathbf{S}(\mathbf{C} a\urcorner v f)(\mathbf{V} \vee v f) f)$ is in the expansion of $x$ by $t$ and $c$ in $s$. Since $l=\mathbf{I} b, f$ satisfies $i$ and $a\left({ }_{\{\mathrm{I} b\}}^{v}\right)$ and so, since $a\left({ }_{\{\mathrm{I} b\}}^{v}\right)$ is an

assigner with respect to $x, t, c$ and $s$ and $f$ is a $z$-level formula of $s$, $\mathbf{S}\left(\mathbf{C} a\left({ }_{\{\mid b\}}^{v}\right) f\right)(\mathbf{V} f) f$ satisfies $i$ and $a\left({ }_{\{\mid b\}}^{v}\right)$. Since $\mathbf{S}\left(\mathbf{C} a\left({ }_{\{I b\}}^{v}\right) f\right)(\mathbf{V} f) f=\mathbf{S}$ (the 
$1^{\text {th }}$ identical of $b$ with respect to $x, t$, and $c$ in $\left.\left.s\right) v(\mathbf{S}(\mathbf{C} a \mathbf{v} v f)(\mathbf{V}\urcorner v f) f\right)$ and (the $1^{\text {th }}$ identical of $b$ with respect to $x, t, c$, and $\left.s\right) \mathrm{I} b$ satisfies $i$ and $a(\underset{\{\mathrm{I} b\}}{v})$, $\mathbf{S} b v(\mathbf{S}(\mathbf{C} a \mathfrak{l} v f)(\mathbf{V}\urcorner v f) f)$ satisfies $i$ and $a\left(\begin{array}{l}v \\ \{\mathbf{I} b\}\end{array}\right)$. Then, since $b \mathbf{I} v$ satisfies $i$ and $a\left(\begin{array}{l}v \\ \{\mathrm{I} b\}\end{array}\right), \mathbf{S}(\mathrm{C} a \boldsymbol{\uparrow} v f)(\mathbf{V} \boldsymbol{v} v) f$ satisfies $i$ and $a\left(\begin{array}{l}v \\ \{\mathrm{I} b\}\end{array}\right)$ and so I $b=k$. Also, since $\mathbf{S} d v(\mathbf{S}(\mathbf{C} a \mathbf{\uparrow} v f)(\mathbf{V}\urcorner v f) f)$ is both in the expansion of $x$ by $t$ and $c$ in $s$ and a $z$-level formula of $s$ and since $a\left(\begin{array}{l}v \\ \left\{I_{d\}}\right\}\end{array}\right)$ is an assigner with respect to $x, t, c$, and $s, \mathbf{S} d v(\mathbf{S}(\mathbf{C} a \boldsymbol{\urcorner} v f)(\mathbf{V} \boldsymbol{\urcorner} v f) f)$ satisfies $i$ and $a\left(\begin{array}{l}v \\ \{\mathrm{I} d\}\end{array}\right)$. Then, since $d \mathbf{I} v$ satisfies $i$ and $a\left(\begin{array}{l}v \\ \{\mathbf{I} d\}\end{array}\right), \mathbf{S}(\mathbf{C} a \mathbf{\eta} v f)(\mathbf{V} \boldsymbol{\gamma} v f) f$ satisfies $i$ and $a\left(\begin{array}{l}v \\ \{\mathbf{I} d\}\end{array}\right)$ and so $\mathbf{I} d=k$. Since $\mathbf{I} a \mathbf{\urcorner} v f=\{l\}, l=\mathbf{I} b, \mathbf{I} a \mathbf{\Upsilon} v(\mathbf{S}(\mathbf{C} a \mathbf{\urcorner} v f)(\mathbf{V} v f) f)=\{k\}, k=\mathbf{I} b, k=\mathbf{I} d$,

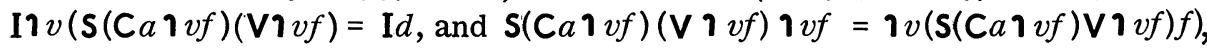
it again follows that $1 v f$ is in $T z+1$. Assume finally that the re is no $l$ in $j$ such that, for any $m$ in $j, f$ satisfies $i$ and $a\left(\begin{array}{l}v \\ \{m\}\end{array}\right)$ just in case $m=l$. It follows that $N g$ both satisfies $i$ and $a$ and is in the expansion of $x$ by $t$ and $c$ in $s$. Since $\mathbf{S}(\mathbf{C} a \boldsymbol{\urcorner} v f)(\mathbf{V}\urcorner v f) \boldsymbol{\urcorner} v f=\boldsymbol{\eta} v(\mathbf{S}(\mathbf{C} a \boldsymbol{\urcorner} v f)(\mathbf{V}\urcorner v f) f)$, it follows by defi-

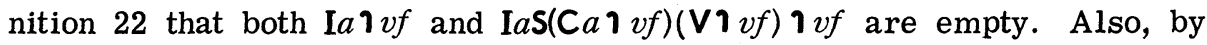
theorem 7, there is no term of $s e$ such that $e \operatorname{II} v(\mathbf{S}(\mathbf{C} a \boldsymbol{l} v f)(\mathbf{V} \boldsymbol{\eta} v f) f)$ is in the expansion of $x$ by $t$ and $c$ in $s$. Hence, IS(C $a$ I $v f)(\mathbf{V} v f) \backslash v f$ is also empty and so $1 v f$ is in $\mathbf{T} z+1$. It follows that (b) of (2) holds and so that the theorem holds.

Notice that the various assumptions made above about $s, x, t, c, i$, and $j$ are not made beyond this point.

Definition 44. If $s$ is a symbol sequence, then

(1) $\mathrm{R} s=$ the remainder of the $1^{\text {th }}$ bisection of the remainder of the $3^{\text {th }}$ bisection of $s$

(2) if $x$ is included in the set of all formulas of $s, x$ is consistent in $s$, and $t$ is an enumerator of the formulas of $s$, then $G x t s=$ the interpreter with respect to $s i$ such that, for any $b$ in the domain of $i$, either $b$ is an individual constant of $s$ and $i(b)=(F$ (the correlate of $x$ in $s) t(R s) s)$ (the correlate of $b$ in $s)$ or $b$ is not an individual constant of $s$ and $i(b)=(\mathbf{F}$ (the correlate of $x$ in $s) t(\mathbf{R} s) s)(b)$.

Notice that, for any symbol sequence $s$ and positive integer $m, \mathbf{R} s(m)=$ the $2 m-1^{\text {th }}$ individual constant of $s$. Since, for any positive integer $m$, the correlate of the $m^{\text {th }}$ individual constant of $s$ in $s=$ the $2 m^{\text {th }}$ individual constant of $s$, it follows that, for any subset $x$ of the set of all formulas of $s$, there are no $c$ in the range of $\mathbf{R} s$ and $f$ in the correlate of $x$ in $s$ such that $c$ is free in $f$.

Theorem 17. If $s$ is a symbol sequence, $x$ is included in the set of all formulas of $s$, and $f$ is a formula of $s$, then

(1) $f$ is in $x$ just in case the correlate of $f$ in $s$ is in the correlate of $x$ in $s$

(2) if $x$ is consistent in $s$, then, for any enumerator of the formulas of $s t$ and any $a$,

(a) $a$ is an assigner for $f$ in UG $x$ ts with respect to $s$ just in case $a$ is an assigner for the correlate of $f$ in $s$ in UF(the correlate of $x$ in $s) t(R s) s$ with respect to $s$ 
(b) if $a$ is an assigner for $f$ in UGxts with respect to $s$, then $f$ satisfies $\mathbf{G} x$ ts and $a$ just in case the correlate of $f$ in $s$ satisfies $\mathbf{F}$ (the correlate of $x$ in $s) t(\mathbf{R} s) s$ and $a$.

This is obvious from definitions 40,43, 44, 28, and 22.

Theorem 18. If $s$ is a symbol sequence, $t$ is a proof sequence in $s, u$ is a finite sequence, the range of $u=$ the set of all $x$ such that, for some $p$ in the range of $t$ and $l$ in the range of $p$, either $l=\left\langle\right.$ show $\left._{s} x\right\rangle$ or $l=x$, $c$ the sequence of individual constants of $s$ in the conjunction for $u$ of $s$, $v$ is a finite sequence, the domain of $v=$ the domain of $c$, the range of $v$ is included in the set of all variables of $s$, there is no $l$ in the domain of $v$ such that either $v(l)$ occurs in the conjunction for $u$ of $s$ or, for some $k$ in the domain of $v, k \neq l$ and $v(k)=v(l), f$ is a correlator, the domain of $f=$ the set of all individual constants of $s$, the range $f$ is included in the set of all individual constants of $s, d$ is a finite sequence, the domain of $d=$ the domain of $c$, and, for any $l$ in the domain of $d, d(l)=f(c(l))$, then the result of properly substituting $d$ for $v$ in (the result of properly substituting $v$ for $c$ in $t$ in $s$ ) in $s$ is a proof sequence in $s$.

The proof is analogous to the one given for theorem 4.

Theorem 19. If $s$ is a symbol sequence, then $f$ is a theorem of $s$ by $L_{s}$ just in case the correlate of $f$ in $s$ is a theorem of $s$ by $L_{s}$.

Assume that $s$ is a symbol sequence. Assume now that $f$ is a theorem of $s$ by $L_{s}$. By definitions $35,34,38,37$, and 39, there is a proof in $s$ by $L_{s}$ $p$ such that either $\langle f\rangle$ is obtainable from $p$ by a direct proof in $s$ or $\langle f\rangle$ is obtainable from $p$ by a universal proof in $s$. By theorem 2 , there is a finite proof sequence in $s r$ such that the last term of $r=p$. By definition 39, $\widetilde{r}\langle<\rangle\rangle$ is a proof sequence in $s$. Let $u$ be a finite sequence such that the range of $u=$ the set of all $x$ such that, for some $q$ in the range of $\widetilde{r} \ll f \gg$ and $l$ in the range of $q$, either $l=$ show $x$ or $l=x$. Also, let $c=$ the sequence of individual constants of $s$ in the conjunction for $u$ of $s$ and let $v$ be a finite sequence such that the domain of $v=$ the domain of $c$, the range of $v$ is included in the set of all variables of $s$, and there is no $l$ in the domain of $v$ such that either $v(l)$ occurs in the conjunction for $u$ of $s$ or, for some $k$ in the domain of $v, k \neq l$ and $v(k)=v(l)$. Also, let $g=$ the function $g$ such that the domain of $g=$ the set of all individual constants of $s$ and $g(c)=$ the correlate of $c$ in $s$ for any $c$ in the domain of $g$. By definitions 40 and 28, $g$ is a correlator. Finally, let $d=$ the finite sequence $d$ such that the domain of $d=$ the domain of $c$ and $d(l)=g(c(l))$ for any $l$ in the domain of $d$. By theorem 18, the result of properly substituting $d$ for $v$ in (the result of properly substituting $v$ for $c$ in $r\langle\langle f\rangle$ in $s$ ) in $s$ is a proof sequence in $s$. Hence, by definitions $40,39,38,37,34$, and 35 , the result of properly substituting $d$ for $v$ in (the result of properly substituting $v$ for $c$ in $f$ in $s$ ) in $s$ is a theorem of $s$ by $L_{s}$. By definitions 40 and 28, it follows that the correlate of $f$ in $s$ is a theorem of $s$ by $L_{s}$. By a similar argument, $f$ is a theorem of $s$ by $L_{s}$ if the correlate of $f$ in $s$ is, Hence, the theorem holds.

Theorem 20. If $s$ is a symbol sequence and $x$ is included in the set of all formulas of $s$, then $x$ is consistent in $s$ just in case the correlate of $x$ in $s$ is consistent in $s$. 
Assume the antecedent. Assume first that $x$ is not consistent in $s$. By definitions 40 and 36, there is a finite sequence $t$ whose range is included in $x$ such that the conjunction for $t$ of $s \rightarrow N \mathrm{~T}_{s}$ is a theorem of $s$ by $\mathrm{L}_{s}$. By theorem 19, the correlate for (the conjunction for $t$ of $s \rightarrow N \mathrm{~T}_{s}$ ) in $s$ is also a theorem of $s$ by $L_{s}$. Let $u=$ the finite sequence $u$ such the domain of $u=$ the domain of $t$ and, for any $l$ in the domain of $u, u(l)=$ the correlate of $t(l)$ in $s$. By definitions 40,36, and 28, the conjunction for $u$ of $s \rightarrow \sim \mathrm{T}_{s}=$ the correlate for (the conjunction for $t$ of $s \rightarrow N \mathrm{~T}_{s}$ ) in $s$. It follows by definitions 40 and 36 that the correlate of $x$ in $s$ is not consistent in $s$. By a similar argument, $x$ is not consistent in $s$ if the correlate of $x$ in $s$ is not. Hence, the theorem holds.

Definition 45. If $s$ is a symbol sequence, then $f$ is a sentence of $s$ just in case $f$ is a formula of $s$ and there is no variable of $s v$ such that $v$ is free in $f$.

Theorem 21. If $s$ is a symbol sequence, $x$ is included in the set of all sentences of $s$, and $x$ is consistent in $s$, then there is an $h$ such that $h$ is an interpreter with respect to $s$ and, for any $f$ in $x, f$ is true with respect to $h$ and $s$.

Assume the antecedent. Let $t$ be an enumerator of the formulas of $s$, let $j=\mathbf{G} x t s$, let $i=\mathbf{F}$ (the correlate of $x$ in $s) t(\mathbf{R} s) s$, and let $a$ be an assigner with respect to the correlate of $x$ in $s, t, \mathbf{R} s$, and $s$. To show that every $f$ in $x$ is true with respect to $j$ and $s$, assume that $f$ is in $x$. By definitions 40, 28 , and 45 and theorem 17, the correlate of $f$ in $s$ is both a sentence of $s$ and in the correlate of $x$ in $s$. Also, by theorem 20, the correlate of $x$ in $s$ is consistent in $s$. Hence, by theorem 16, the correlate of $f$ in $s$ satisfies $i$ and $a$ just in case the result of properly substituting (the sequence of individual constants of $s$ for the correlate of $f$ in $s$ with respect to $a$, the correlate of $x$ in $s, t$, and $\mathrm{R} s$ ) for (the sequence of variables of $s$ free in the correlate of $f$ in $s$ ) in the correlate of $f$ in $s$ in $s$ is in the expansion of the correlate of $x$ in $s$ by $t$ and $\mathbf{R} s$ in $s$; that is, just in case the correlate of $f$ in $s$ is in this expansion. Since the correlate of $x$ in $s$ is included in the expansion of the correlate of $x$ in $s$ by $t$ and $\mathbf{R} s$ in $s$, it follows that the correlate of $f$ in $s$ satisfies $i$ and $a$; that is, by theorem 17, $f$ satisfies $j$ and $a$. But, since no variable of $s$ is free in $f, f$ satisfies $j$ and $a$ for any assigner for $f$ in $\mathrm{U} j a$ and so for any proper assigner for $f$ in $\mathrm{U}_{j} a$; that is, by definition $25, f$ is true with respect to $j$ and $s$. Hence, the theorem holds.

Definition 46. If $s$ is a symbol sequence and $f$ is a formula of $s$, then

(1) the $o^{\text {th }}$ closure of $f$ in $s=f$

(2) for any $m$, if $m$ is in the domain of the sequence of variables of $s$ free in $f$, then the $m^{\text {th }}$ closure of $f$ in $s=\ll N_{s}<v_{s}$ the $m^{\text {th }}$ variable of $s$ free in $f<\mathbf{E}_{s}$ the $m^{\text {th }}$ variable of $s$ free in $f \gg \gg \wedge_{s}$ the result of properly substituting $<\boldsymbol{1}_{s}$ the $m^{\text {th }}$ variable of $s$ free in $f<N_{s}<\mathbf{I}_{s}$ the $m^{\text {th }}$ variable of $s$ free in $f$ the $m^{\text {th }}$ variable of $s$ free in $f \gg$ for the $m^{\text {th }}$ variable of $s$ free in $f$ in the $m-1^{\text {th }}$ closure of $f$ in $s$ in $s>v_{s} \ll v_{s}$ the $m^{\text {th }}$ variable of $s$ free in $f<\mathbf{E}_{s}$ the $m^{\text {th }}$ variable of $s$ free in $f \gg \wedge_{s}\left\langle\wedge_{s}\right.$ the $m^{\text {th }}$ variable of $s$ free in $f$ the $m-1^{\text {th }}$ closure of $f$ in $s \gg$

(3) either no variable of $s$ is free in $f$ and the closure of $f$ in $s=$ the 
$O^{\text {th }}$ closure of $f$ in $s$ or some variable of $s$ is free in $f$ and the closure of $f$ in $s=$ the (the greatest member of the domain of the sequence of variables of $s$ free in $f)^{\text {th }}$ closure of $f$ in $s$.

Theorem 22. If $s$ is a symbol sequence and $f$ is a formula of $s$, then

(1) $f$ is valid with respect to $s$ just in case the closure of $f$ in $s$ is valid with respect to $s$

(2) $f$ is a theorem of $s$ by $L_{s}$ just in case the closure of $f$ in $s$ is a theorem of $s$ by $L_{s}$.

To establish (1), it is sufficient to show that

(a) $f$ is valid with respect to $s$ just in case the $0^{\text {th }}$ closure of $f$ in $s$ is valid with respect to $s$

(b) for any $m$ in the domain of the sequence of variables of $s$ free in $f$, if $f$ is valid with respect to $s$ just in case the $m-1^{\text {th }}$ closure of $f$ in $s$ is valid with respect to $s$, then $f$ is valid with respect to $s$ just in case the $m^{\text {th }}$ closure of $s$ in $f$ is valid with respect to $s$.

It is obvious that (a) holds. To show (b), assume that $m$ is in the domain of the sequence of variables of $s$ free in $f$ and that $f$ is valid with respect to $s$ just in case the $m-I^{\text {th }}$ closure of $f$ in $s$ is. Let $v=$ the $m^{\text {th }}$ variable of $s$ free in $f$, let $g=$ the $m-1^{\text {th }}$ closure of $f$ in $s$, and let $h=$ the result of properly substituting $\mathbf{1} v \sim v \mathbf{I} v$ for $v$ in $g$ in $s$. By definition 46 , the $m^{\text {th }}$ chosure of $f$ in $s=\langle N \vee v \mathbf{E} v \wedge h\rangle \vee\langle\vee v \mathbf{E} v \wedge \wedge v g\rangle$. Since $g \leftrightarrow\langle\wedge \vee v \mathrm{E} v \wedge g\rangle \vee\langle\vee v \mathrm{E} v \wedge g\rangle$ is valid with respect to $s, g$ is valid with respect to $s$ just in case $\langle N \vee v \mathrm{E} v \wedge g\rangle \vee\langle\vee v \mathrm{E} v \wedge g\rangle$ is; that is, since $N \vee v E v \rightarrow\langle g \leftrightarrow h\rangle$ is valid with respect to $s$, just in case $\langle N \vee v \mathrm{E} v \wedge h\rangle_{\vee}\langle\vee v \mathrm{E} v \wedge g\rangle$ is. Assume first that $g$ is valid with respect to $s$. Since $v$ is free neither in $N \vee v \mathrm{E} v \wedge h$ nor in $\vee v \mathrm{E} v$ and since, for any formulas of $s a, e$, and $g$ such that $v$ is free in neither $a$ nor $e$, $a \vee\langle e \wedge \wedge v g\rangle$ is valid with respect to $s$ if $a \vee\langle e \wedge g\rangle$ is, it follows that $\langle N \vee v \mathrm{E} v \wedge h\rangle \vee\langle\mathrm{V} v \mathrm{E} v \wedge \wedge v g\rangle$ is valid with respect to $s$. Assume on the other hand that $\langle N \vee v \mathrm{E} v \wedge h\rangle \vee\langle\vee v \mathrm{E} v \wedge \wedge v g\rangle$ is valid with respect to $s$. Since $\vee v E v \wedge \wedge v g \rightarrow g$ is valid with respect to $s$, it follows that $\langle N \vee v \mathrm{E} v \wedge h\rangle \vee\langle\vee v \mathrm{E} v \wedge g\rangle$ is; that is, $g$ is. Hence, (b) holds and so (1) holds. The reasoning for (2) is the same as that for (1), but with respect to theoremhood instead of with respect to validity.

Theorem 23. If $s$ is a symbol sequence, $f$ is a formula of $s$, and $f$ is valid with respect to $s$, then $f$ is a theorem of $s$ by $L_{s}$.

Assume the antecedent. By theorem 22, the closure of $f$ in $s$ is valid with respect to $s$. Also, by definitions 45 and 46 and theorem 21, if $\{N$ the closure of $f$ in $s\}$ is consistent in $s$, then, for some interpreter with respect to $s h, N$ the closure of $f$ in $s$ is true with respect to $h$ and $s$. Since there is no such $h,\{N$ the closure of $f$ in $s\}$ is not consistent in $s$ and so implies the closure of $f$ in $s$; that is, since the empty set and $<N$ the closure of $f$ in $s>$ are the only non-repeating finite sequences whose ranges are included in $\{N$ the closure of $f$ in $s\}$, either $\mathbf{T}_{s} \rightarrow$ the closure of $f$ in $s$ or $N$ the closure of $f$ in $s \rightarrow$ the closure of $f$ in $s$ is a theorem of $s$ by $L_{s}$. Since both $T_{s}$ and $\langle N c \rightarrow c\rangle \rightarrow c$ are theorems of $s$ by $L_{s}$ for any formula of $s c$, the closure of $f$ in $s$ is then a theorem of $s$ by $L_{s}$; hence, by theorem 22, $f$ is a theorem of $s$ by $L_{s}$. 
Theorem 23 has some interesting corollaries.

Theorem 24. If $s$ is a symbol sequence, $f$ is a formula of $s, v$ is a variable of $s$, and, for any $i$, if $i$ is an interpreter with respect to $s$ and $U i=$ the empty set, then $f$ is true with respect to $i$ and $s$, then $\left\langle N_{s}\left\langle V_{s} v\left\langle E_{s} v \gg\right\rangle \rightarrow{ }_{s} f\right\rangle\right.$ is a theorem of $s$ by $L_{s}$.

Assume the antecedent. Obviously, for any interpreter with respect to $s i$, either $\mathrm{U} i$ is empty and $N \vee v \mathrm{E} v$ is true with respect to $i$ and $s$ or $\mathrm{U} i$ is not empty and $\mathrm{V} v \mathrm{E} v$ is true with respect to $i$ and $s$. Then, since $N \vee v E v$ is a sentence of $s, N \vee v \mathrm{E} v \rightarrow f$ is valid with respect to $s$; hence, by theorem 23, $N \vee v \mathrm{E} v \rightarrow f$ is a theorem of $s$ by $L_{s}$.

Theorem 25. If $s$ is a symbol sequence, $f$ is a formula of $s, v$ is a variable of $s$, and $d=\left\langle\left\{\left\langle N_{s}\left\langle V_{s} v\left\langle\mathrm{E}_{s} v\right\rangle\right\rangle\right\} \mathrm{L}_{s}(2) \mathrm{L}_{s}(3) \mathrm{L}_{s}(4)\right\rangle\right.$, then $f$ is a theorem of $s$ by $d$ just in case, for any $i$, if $i$ is an interpreter with respect to $s$ and $\mathrm{U} i=$ the empty set, then $f$ is true with respect to $i$ and $s$.

Assume the antecedent. Since, for any interpreter with respect to $s i$, $\mathrm{U} i$ is empty just in case $N \vee v E v$ is true with respect to $i$ and $s$, we have the consequent by theorems 3 and 24 .

Theorem 26. If $s$ is a symbol sequence, $f$ is a formula of $s, v$ is a variable of $s$, and, for any $i$, if $i$ is an interpreter with respect to $s$ and $U i \neq$ the empty set, then $f$ is true with respect to $i$ and $s$, then $\left\langle\left\langle\mathrm{V}_{s} v\left\langle\mathrm{E}_{s} v \gg \rightarrow{ }_{s} f\right\rangle\right.\right.$ is a theorem of $s$ by $\mathrm{L}_{s}$.

The proof is analogous to the one given for theorem 24 .

Theorem 27. If $s$ is a symbol sequence, $f$ is a formula of $s, v$ is a variable of $s$, and $d=\left\langle\left\{\left\langle\mathrm{V}_{s} v\left\langle\mathrm{E}_{s} v\right\rangle\right\} \mathrm{L}_{s}(2) \mathrm{L}_{s}(3) \mathrm{L}_{s}(4)\right\rangle\right.$, then $f$ is a theorem of $s$ by $d$ just in case, for any $i$, if $i$ is an interpreter with respect to $s$ and $\mathrm{U} i \neq$ the empty set, then $f$ is true with respect to $i$ and $s$.

The proof is analogous to the one given for theorem 25 .

\section{THEORIES AND RELATED CONCEPTS}

A (non-logical) theory can be understood as a deductive system based on logic in which more than the theorems of logic (particularly existence theorems) are provable.

Definition 47. If $s$ is a symbol sequence, then, $x$ is a theory in $s$ just in case $x$ satisfies the following conditions:

(1) $x$ is a deductive system in $s$

(2) for any $m$ in the domain of $\mathrm{L}_{s}, \mathrm{~L}_{s}(m)$ is included in $x(m)$

(3) for some $f, f$ is a theorem of $s$ by $x$ and $f$ is not a theorem of $s$ by $L_{s}$.

Theories are generally held to have subject matters; set theories are about sets, number theories are about numbers, and so on. Yet, it is often the case that the members of various very different universes of discourse behave in the way that the entities that the theory is about behave; that is, all of the theorems of the theory hold in these universes under one or another interpretation. Any such universe can be understood as a subject matter for that theory. 
Definition 48. If $s$ is a symbol sequence and $t$ is a theory in $s$, then $x$ is a subject matter for $t$ with respect to $s$ just in case there is an interpreter with respect to $s i$ such that $x=U i$ and, for any theorem of $s$ by $t f$, $f$ is true with respect to $i$ and $s$.

Notice that only consistent theories have subject matters. Notice also that a subject matter for a theory $t$ can be used to define the entities that $t$ is about. For instance, if $s$ is a symbol sequence, $t$ is both a theory in $s$ and one or another axiomatization of set theory, and $x$ is a subject matter for $t$ with respect to $s$, then one can say that $y$ is a set with respect to $x$ just in case $y$ is in $x$.

In the first chapter of book 4 of the collection of Aristotelean writings to which Andronicus of Rhodes arbitrarily attached a synonym of the word 'Metaphysics', the philosopher says ${ }^{6}$

There is a science which investigates being as being and the attributes which belong to this in virtue of its own nature. Now this is not the same as any of the so-called special sciences, for none of them treats universally of being as being.

This passage strongly suggests an exact definition of what a metaphysical system is. Suppose that we define an existence formula to be any formula which is either a statement that so and so exists or a self identity statement or a non-vacuous existential generalization or the negation of a non-vacuous universal generalization. We may then define an ontology to be a set of existence formulas. Moreover, given an ontology $a$, the metaphysical system determined by $a m$ can be understood as that axiomatic extension of logic which investigates all and only the consequences of existing according to $a$; that is, $m$ is the result of adding the axioms $a$ to logic.

Definition 49. If $s$ is a symbol sequence, then

(1) $f$ is an existence formula of $s$ just in case $f$ satisfies one of the following conditions:

(a) for some term of $s t, f=\left\langle\mathrm{E}_{s} t\right\rangle$

(b) for some term of $s t, f=\left\langle\mathbf{I}_{s} t t\right\rangle$

(c) for some variable of $s v$ and formula of $s g, v$ is free in $g$ and $f=\left\langle\mathrm{V}_{s} v g\right\rangle$

(d) for some variable of $s v$ and formula of $s g, v$ is free in $g$ and $f=\left\langle N_{s}\left\langle\wedge_{s} v g\right\rangle\right\rangle$

(2) $x$ is an ontology in $s$ just in case $x$ is included in the set of all existence formulas of $s$

(3) if $a$ is an ontology in $s$, then the metaphysical system in $s$ determined by $a=\left\langle a \mathrm{~L}_{s}(2) \mathrm{L}_{s}(3) \mathrm{L}_{s}(4)\right\rangle$.

Metaphysical systems, the ontology of a theory, and the metaphysical system of a theory can now be defined in an obvious way.

Definition 50. If $s$ is a symbol sequence, then

(1) $x$ is a metaphysical system in $s$ just in case, for some ontology in $s a, x=$ the metaphysical system in $s$ determined by $a$

(2) if $t$ is a theory in $s$, then

(a) the ontology of $t$ in $s=$ the set of all existence formulas of $s f$ such that $f$ is a theorem of $s$ by $t$ 
(b) the metaphysical system of $t$ in $s=$ the metaphysical system in $s$ determined by the ontology of $t$ in $s$.

Notice that logic is the metaphysical system determined by the empty set. Notice also that, for any symbol sequence $s$ and theory in $s t$, if, for some term of $s u, \mathrm{E} u$ is a theorem of $s$ by $t$, then there is a metaphysical system in $s m$ such that the set of all theorems of $s$ by $t=$ the set of all theorems of $s$ by $m$. Given such a theory $t$, let $a=$ the set of all $f$ such that, for some theorem of $s$ by $t u$ and variable of $s v, v$ is not free in $u$ and $f=$ $\vee v\langle\mathrm{E} v \wedge u\rangle$ and let $m=\left\langle a \mathrm{~L}_{s}(2) \mathrm{L}_{s}(3) \mathrm{L}_{s}(4)\right\rangle$. If $u$ is a theorem of $s$ by $t$, then, for some variable of $s$ not free in $u v, \vee v\langle\mathrm{E} v \wedge u\rangle$ is a theorem of $s$ by $m$ and so $u$ is a theorem of $s$ by $m$. Also, if $f$ is in $m(1)$, then, for some theorem of $s$ by $t u$ and variable of $s v$ not free in $u, f=\vee v\langle\mathrm{E} v \wedge u\rangle$ and so $f$ is a theorem of $s$ by $t$. Also, for any positive integer $n$ greater than 1 and not greater than $4, m(n)$ is included in $t(n)$. Hence, every theorem of $s$ by $m$ is also a theorem of $s$ by $t$. Also, $m$ is obviously a metaphysical system in $s$. It follows that metaphysical systems can be rather strong. However, since, for any metaphysical system $m$, there exists a deductive system which is not a metaphysical system whose theorems are those of $m$, it also follows that metaphysical systems are avoidable.

Given a symbol sequence $s$, a theory in $s t$, an interpreter with respect to $s i$, a finite subset of $U i x$, and a non-empty finite sequence of theorems of $s$ by $t u$, the degree of confirmation of $t$ with respect to $i, x, u$, and $s$ can be understood as the mean degree of truth of the terms of $u$ in $x$ with respect to $i$ and $s$. This concept can easily be extended to empty and denumerably infinite $u$.

Definition 51. If both either $s$ is a finite sequence or $s$ is denumerably infinite sequence and the range of $s$ is included in the set of all real numbers, then

(1) $s$ converges to $x$ just in case $x$ is a real number and one of the following conditions is satisfied:

(a) for some $m$ in the domain of $s, s(m)=x$ and there is no $n$ in the domain of $s$ such that $m$ is smaller than $n$

(b) both there is an $n$ in the domain of $s$ such that $m$ is smaller than $n$ for any $m$ in the domain of $s$ and, for any positive real number $r$, there is an $m$ in the domain of $s$ such that, for any $n$ in the domain of $s$, if $m$ is smaller than $n$, then $x-r$ is smaller than $s(n)$ and $s(n)$ is smaller than $x+r$

(2) the limit of $s=$ the $x$ satisfying one of the following conditions:

(a) $s$ converges to $x$

(b) $x=0$ and there is no $y$ such that $s$ converges to $y$

(3) the sum of $s=$ the limit of the $t$ satisfying the following conditions:

(a) $t$ is a function

(b) the domain of $t=$ the domain of $s$

(c) for any $m$ in the domain of $t$, either $m=1$ and $t(m)=s(m)$ or $m$ is greater than 1 and $t(m)=t(m-1)+s(m)$.

Definition 52. If $s$ is a symbol sequence, $t$ is a theory in $s, i$ is an interpreter with respect to $s, x$ is a finite subset of $U i$, either $u$ is a finite sequence or $u$ is a denumerably infinite sequence, and the range of $u$ is in- 
cluded in the set of all theorems of $s$ by $t$, then the degree of confirmation of $t$ with respect to $i, x, u$, and $s=$ the limit of the $y$ satisfying the following conditions:

(a) $y$ is a function

(b) the domain of $y=$ the domain of $u$

(c) for any $m$ in the domain of $y, y(m)=$ the $z$ such that, for some $l, z=l / m$ and $l=$ the sum of the $w$ satisfying the following conditions:

(1) $w$ is a function

(2) the domain of $w=$ the domain of $u$ cut off at the $m+1^{\text {th }}$

term

(3) for any $k$ in the domain of $w, w(k)=$ the degree of truth of $u(k)$ in $x$ with respect to $i$ and $s$.

Also, given a symbol sequence $s$, a theory in $s t$, and a non-empty finite sequence of formulas of $s u$, the explanatory power of $t$ with respect to $u$ and $s$ can be understood as the ratio of the number of terms of $u$ which are theorems of $s$ by $t$ to the length of $u$. The concept can be extended to empty and denumerably infinite $u$ just as degree of confirmation was.

Definition 53. If $s$ is a symbol sequence, $t$ is a theory in $s$, either $u$ is a finite sequence or $u$ is a denumerably infinite sequence, and the range of $u$ is included in the set of all formulas of $s$, then the explanatory power of $t$ with respect to $u$ and $s=$ the limit of the $y$ satisfying the following conditions:

(1) $y$ is a function

(2) the domain of $y=$ the domain of $u$

(3) for any $m$ in the domain of $y, y(m)=$ the $z$ such that, for some $l$, $z=l / m$ and $l=$ the number of members of the set of all $k$ in the domain of $u$ cut off at the $m+1^{\text {th }}$ term such that $u(k)$ is a theorem of $s$ by $t$.

Finally, given a symbol sequence $s$, a theory in $s t$, a set of formulas of $s f$, and a non-empty finite sequence of theorems of $s$ by $t u$, the degree of deductive simplicity of $t$ with respect to $f, u$, and $s$ can be understood as the ratio of the number of terms of $u$ which are in $f$ to the length of $u$. As before, the concept can be extended to empty and denumerably inf inite $u$.

Definition 54. If $s$ is a symbol sequence, $t$ is a theory in $s, f$ is included in the set of all formulas of $s$, either $u$ is a finite sequence or $u$ is a denumerably infinite sequence, and the range of $u$ is included in the set of all theorems of $s$ by $t$, then the degree of deductive simplicity of $t$ with respect to $f, u$, and $s=$ the limit of the $y$ satisfying the following conditions:

(1) $y$ is a function

(2) the domain of $y=$ the domain of $u$

(3) for any $m$ in the domain of $y, y(m)=$ the $z$ such that, for some $l$, $z=l / m$ and $l=$ the number of members of the set of all $k$ in the domain of $u$ cut off at the $m+1^{\text {th }}$ term such that $u(k)$ is in $f$.

The concepts of definitions 52 through 54 can be concocted into a concept of degree of adequacy for theories. Suppose that we define the weight of a finite or denumerably infinite sequence as in definition 55 .

Definition 55. If either $s$ is a finite sequence or $s$ is a denumerably infinite sequence, then the weight of $s=$ the limit of the $t$ satisfying the following conditions: 
(1) $t$ is a function

(2) the domain of $t=$ the domain of $s$

(3) for any $m$ in the domain of $t, t(m)=(2 m-1) / 2 m$.

Given a symbol sequence $s$, a theory in $s t$, an interpreter with respect to $s i$, a finite subset of $U_{i} x$, finite or denumerably infinite sequences $u, v$, and $w$, and a set of formulas of $s f$, if $u$ and $w$ are sequences of theorems of $s$ by $t$ and $v$ is a sequence of formulas of $s$, then the degree of adequacy of $t$ with respect to $i, x, u, v, f, w$, and $s$ can be understood as the weighted mean of the degree of confirmation, explanatory power, and degree of deductive simplicity of $t$ with respect to the appropriate sets and with the weights of $u, v$, and $w$ as weights if $t$ is consistent in $s$ and as $O$ if $t$ is not consistent in $s^{7}$.

Definition 56. If $s$ is a symbol sequence, $t$ is a theory in $s, i$ is an interpreter with respect to $s, x$ is a finite subset of $U i$, either $u$ is a finite sequence or $u$ is a denumerably infinite sequence, the range of $u$ is included in the set of all theorems of $s$ by $t$, either $v$ is a finite sequence or $v$ is a denumerably infinite sequence, the range of $v$ is included in the set of all formulas of $s, f$ is included in the set of all formulas of $s$, either $w$ is a finite sequence or $w$ is a denumerably infinite sequence, and the range of $w$ is included in the set of all theorems of $s$ by $t$, then the degree of adequacy of $t$ with respect to $i, x, u, v, f, w$, and $s=$ the $y$ satisfying one of the following conditions:

(1) $y=0$ and either one of $u, v$, and $w$ are all empty or every formula of $s$ is a theorem of $s$ by $t$

(2) one of $u, v$, and $w$ is not empty, there is a formula of $s g$ such that $g$ is not a theorem of $s$ by $t$, and, for some $k, l, m, p, q$, and $r$,

(a) $k=$ the degree of confirmation of $t$ with respect to $i, x, u$, and $s$

(b) $l=$ the explanatory power of $t$ with respect to $v$ and $s$

(c) $m=$ the degree of deductive simplicity of $t$ with respect to $f$, $w$, and $s$

(d) $p=$ the weight of $u$

(e) $q=$ the weight of $v$

(f) $r=$ the weight of $w$

(g) $y=((k p+l q)+m r) /((p+q)+r)$.

\section{CONCRETENESS AND RELATED CONCEPTS}

Various philosophers hold something like the position that expressions can be divided into those which are more or less elementary and those which are more or less abstract in some sense ${ }^{8}$. In this paper, it is assumed that elementaryness depends on both an interpreter $i$ and an arbitrary set $x$ and amounts to concreteness with respect to $i$ and $x$.

Definition 57. If $s$ is a symbol sequence, $e$ is an expression of $s, x$ is a set, and $i$ is an interpreter with respect to $s$, then

(1) if either $e$ is a variable of $s$ or $e=N_{s}$ or $e$ is a standard binary connective of $s$ or $e$ is a variable binder of $s$ or $e$ is an auxiliary symbol of $s$, then $e$ is concrete in $x$ with respect to $i$ and $s$ just in case $e=e$ 
(2) if $e$ is an individual constant of $s$, then $e$ is concrete in $x$ with respect to $i$ and $s$ just in case $i(e)$ is a non-empty subset of $x$

(3) for any positive integer $m$,

(a) if $e$ is an $m$-place operation symbol of $s$, then $e$ is concrete in $x$ with respect to $i$ and $s$ just in case, for any $m$-term sequence whose range is included in the set of all terms of $s t$ and assigner for $\langle e\rangle t$ in $\mathrm{U} i$ with respect to $s a$, if, for any $j$ in the domain of $t$, the interpretation of $t(j)$ with respect to $i$ and $a$ is a non-empty subset of $x$, then the interpretation of $\langle e\rangle_{t}$ with respect to $i$ and $a$ is a non-empty subset of $x$

(b) if $e$ is an $m$-place predicate of $s$, then $e$ is concrete in $x$ with respect to $i$ and $s$ just in case $i(e)$ is included in the set of all $m$-term sequences whose ranges are included in $x$

(4) if $e$ is not a standard symbol of $s$, then $e$ is concrete in $x$ with regard to $i$ and $s$ just in case, for any $m$ in the domain of $e, e(m)$ is concrete in $x$ with respect to $i$ and $s$.

Definition 58. If $s$ is a symbol sequence, $e$ is an expression of $s, x$ is a set, and $i$ is an interpreter with respect to $s$, then $e$ is abstract in $x$ with respect to $i$ and $s$ just in case $e$ is not concrete in $x$ with respect to $i$ and $s$.

In other words, an expression is concrete in a set $x$ with respect to an interpreter $i$ just in case $i$ interprets the descriptive constants of $e$ which are not variable binders within $x$ and is abstract in $x$ with respect to $i$ otherwise ${ }^{9}$.

The concreteness concept of definition 57 can be used to characterize applications of theories.

Definition 59. If $s$ is a symbol sequence, $t$ is a theory in $s, x$ is a set, and $i$ is an interpreter with respect to $s$, then $f$ is an application of $t$ in $x$ with respect to $i$ and $s$ just in case $f$ satisfies the following conditions:

(1) $f$ is included in the set of all theorems of $s$ by $t$

(2) no theorem of $s$ by $\mathrm{L}_{s}$ is in $f$

(3) every $g$ in $f$ is concrete in $x$ with respect to $i$ and $s$.

In other words, an application of a theory is a set of concrete non-logical consequences of the theory. We assume here (as, incidentally, we did throughout section 7) that all desirable factual statements, assumptions, idealizations, and so on are theorems of the concerned theory.

Given a symbol sequence $s$, a theory in set $t$, a set $x$, and an interpreter with respect to $s t, t$ can be called significant in $x$ with respect to $i$ and $s$ just in case $t$ has a non-empty application in $x$ with respect to $i$ and $s$.

Definition 60. If $s$ is a symbol sequence, $t$ is a theory in $s, x$ is a set, and $i$ is an interpreter with respect to $s$, then $t$ is significant in $x$ with respect to $i$ and $s$ just in case there is an $f$ satisfying the following conditions:

(1) $f$ is not empty

(2) $f$ is an application of $t$ in $x$ with respect to $i$ and $s$.

This concept of significance is rather weak in that it allows many insignificant theories to be made significant through the me re addition of an appropriate concrete definition. Nevertheless, the policy of not calling such definitional extensions significant is a difficult one to justify. 


\section{THE INTERPRETATION OF EXPRESSIONS}

Some of the philosophers who adopt something like the distinction between elementary and abstract expressions in addition adopt something like the position that only elementary expressions require interpretation since abstract expressions are given meanings by certain deductive relations which they bear to elementary expressions ${ }^{10}$.

In this paper, it is assumed that all expressions are given meanings by interpretation and without dependence upon any of the many different theories in which some expressions may be used.

Given a symbol sequence $s$, an expression of $s e$, and an interpreter with respect to $s i$, the denotation of $e$ with respect to $i$ and $s$ can be understood as $e$ if $e$ is a standard symbol of $s$ which is not a descriptive constant of $s$, with the interpretation of $e$ if $e$ is a descriptive constant of $s$, and with the appropriate combination of the denotations of the constituents of $e$ if $e$ is not a standard symbol of $s$.

Definition 61. If $s$ is a symbol sequence, $e$ is an expression of $s$, and $i$ is an interpreter with respect to $s$, then

(1) if $e$ is a standard symbol of $s$ and $e$ is not a descriptive constant of $s$, then the denotation of $e$ with respect to $i$ and $s=e$

(2) if $e$ is a descriptive constant of $s$, then the denotation of $e$ with respect to $i$ and $s=i(e)$

(3) if $e$ is not a standard symbol of $s$, then the denotation of $e$ with respect to $i$ and $s=$ the $t$ satisfying the following conditions:

(a) $t$ is a finite sequence

(b) the domain of $t=$ the domain of $e$

(c) for any $m$ in the domain of $t, t(m)=$ the denotation of $e(m)$ with respect to $i$ and $s$.

Given a symbol sequence $s$, an expression of $s e$, and a set of interpreters with respect to $s i$, the meaning of $e$ with respect to $i$ and $s$ can be understood as the set of all ordered pairs whose first terms are members of $i$ and whose second terms are the denotations of $e$ with respect to their first terms and $s$.

Definition 62. If $s$ is a symbol sequence, $e$ is an expression of $s, i$ is a set, and every $j$ in $i$ is an interpreter with respect to $s$, then the meaning of $e$ with respect to $i$ and $s=$ the $x$ satisfying the following conditions:

(1) $x$ is a function

(2) the domain of $x=i$

(3) for any $j$ in the domain of $x, x(j)=$ the denotation of $e$ with respect to $j$ and $s$.

Although the denotations of definition 61 and the meanings of definition $62^{11}$ are not relativized to formulas, it may sometimes be desirable to attend only to those denotations and meanings which fulfill all $g$ in a particular set of formulas. Such denotations and meanings can be called admissible with respect to the appropriate sets.

Definition 63. If $s$ is a symbol sequence and $f$ is included in the set of all formulas of $s$, then 
(1) if $i$ is an interpreter with respect to $s$, then $i$ is admissible with respect to $f$ and $s$ just in case, for any $g$ in $f, g$ is true with respect to $i$ and $s$

(2) if $e$ is an expression of $s$, then

(a) $x$ is an admissible denotation of $e$ with respect to $f$ and $s$ just in case, for some interpreter with respect to $s i, i$ is admissible with respect to $f$ and $s$ and $x=$ the denotation of $e$ with respect to $i$ and $s$.

(b) $x$ is an admissible meaning of $e$ with respect to $f$ and $s$ just in case, for some set $i, x=$ the meaning of $e$ with respect to $i$ and $s$ and, for any $j$ in $i, j$ is an interpreter with respect to $s$ and $j$ is admissible with respect to $f$ and $s$.

Given a symbol sequence $s$ and a subset of the set of all formulas of $s f$, any set within which some interpreter admissible with respect to $f$ and $s$ assigns interpretations can be understood as a model for $f$ in $s$.

Definition 64. If $s$ is a symbol sequence and $f$ is included in the set of all formulas of $s$, then $x$ is a model for $f$ in $s$ just in case, for some interpreter with respect to $s i, i$ is admissible with respect to $f$ and $s$ and $x=U i$.

Suppose now that the degrees of truth of all $g$ in a particular set of formulas have been established with respect to certain sets of the relevant kinds and that it is desirable to attend only to those denotations and meanings which lead to these results. A relation of admissibility for such denotations and meanings can also be defined.

Definition 65. If $s$ is a symbol sequence, $f$ is included in the set of all formulas of $s, i$ is an interpreter with respect to $s$, and $x$ is a finite subset of $\mathrm{U} i$, then

(1) if $j$ is an interpreter with respect to $s$, then $j$ is admissible with respect to $f, i, x$, and $s$ just in case $\mathrm{U} j$ is finite and, for any $g$ in $f$, the degree of truth of $g$ in $U j$ with respect to $j$ and $s=$ the degree of truth of $g$ in $x$ with respect to $i$ and $s$

(2) if $e$ is an expression of $s$, then

(a) $z$ is an admissible denotation of $e$ with respect to $f, i, x$, and $s$ just in case, for some interpreter with respect to $s j, j$ is admissible with respect to $f, i, x$, and $s$ and $z=$ the denotation of $e$ with respect to $j$ and $s$

(b) $z$ is an admissible meaning of $e$ with respect to $f, i, x$, and $s$ just in case, for some set $j, z=$ the meaning of $e$ with respect to $j$ and $s$ and, for any $k$ in $j, k$ is an interpreter with respect to $s$ and $k$ is admissible with respect to $f, i, x$, and $s$.

Given a symbol sequence $s$, a subset of the set of all formulas of $s f$, an interpreter with respect to $s i$, and a finite subset of $U i x$, any set within which some interpreter admissible with respect to $f, i, x$, and $s$ assigns interpretations can be understood as a model for $f$ by $i$ and $x$ in $s$.

Definition 66. If $s$ is a symbol sequence, $f$ is included in the set of all formulas of $s, i$ is an interpreter with respect to $s$, and $x$ is a finite subset of $\mathrm{U} i$, then $y$ is a model for $f$ by $i$ and $x$ in $s$ just in case, for some interpreter with respect to $s j, j$ is admissible with respect to $f, i, x$, and $s$ and $y=\mathbf{U} j$.

Denotations and meanings admissible in the sense of leading to a certain degree of confirmation for a theory or to a certain degree of adequacy 
for a theory could also be defined with respect to the relevant sets although they will not be defined here. All of this establishes the fact that there is a good sense in which the expressions of a symbol sequence have more than one kind of denotation and more than one kind of meaning.

\section{NOTES}

1. See, for instance, the modification and extension of the semantics of A. Tarski used by R. Montague and D. Kalish in 'Remarks on descriptions and natural deduction' (Archiv für Mathematische Logik und Grundlagenforschung, v. 3, 1957).

2. The resulting semantics is nevertheless a descendant of the semantics used in the paper cited in the previous note. The deductive system $L_{s}$ of section 4 and some of the proofs of sections 5 and 6 also have ancestors in that paper.

3. As far as the author knows, the idea that $O$-place variable binders bind all free variables in their scopes is due to Richard Montague.

4. Assume that $s$ is a symbol sequence. Suppose that we call $a$ an assigner in a set $x$ with respect to $s$ just in case, for any term or formula of $s t$, $a$ is an assigner for $t$ in $x$ with respect to $s$. Obviously, a formula of $s f$ is valid with respect to $s$ just in case, for any interpreter with respect to $s i$ and assigner in $\mathrm{U} i$ with respect to $s a, f$ satisfies $i$ and $a$. Now, for any interpreter with respect to $s i$ and assigner in $U i$ with respect to $s a$, let $\mathbf{E} i a=$ the function $f$ such that the domain of $f=$ the set of all formulas of $s$ and, for any $g$ in the domain of $a$, either $g$ satisfies $i$ and $a$ and $f(g)=1$ or $g$ does not satisfy $i$ and $a$ and $f(g)=0$. Also, for any interpreter with respect to $s i$ and natural number $k$, let $\mathbf{T} i k=$ the set of all $k$-level terms of $s t$ such that

(1) for any variable of $s v$ and assigner in $U i$ with respect to $s a$, if $v$ is not free in $t$, then either $U i$ is empty and the interpretation of $t$ with respect to $i$ and $a=$ the interpretation of $t$ with respect to $i$ and $a$ ( $\begin{aligned} & v \\ & \text { the }\end{aligned}$ empty set) or $\mathbf{U} i$ is not empty and, for any $m$ in $\mathbf{U} i$, the interpretation of $t$ with respect to $i$ and $a=$ the interpretation of $t$ with respect to $i$ and $a\left(\begin{array}{ll}v \\ \{m\}\end{array}\right)$

(2) for any $k$-level term of $s b$, variable of $s v$, and assigner in $\mathrm{U} i$ with respect to $s a$, if either $U i$ is empty or the interpretation of $b$ with respect to $i$ and $a$ is not empty, then the interpretation of the result of properly substituting $b$ for $v$ in $t$ in $s$ with respect to $i$ and $a=$ the interpretation of $t$ with respect to $i$ and $a($ the interpretation of $b$ with respect to $i$ and $a)$

(3) for any $k$-level terms of $s b$ and $c$ and assigner in $U i$ with respect to $s a$, if the interpretation of $b$ with respect to $i$ and $a=$ the interpretation of $c$ with respect to $i$ and $a$, then the interpretation of the result of properly substituting $b$ for $c$ in $t$ in $s$ with respect to $i$ and $a=$ the interpretation of $t$ with respect to $i$ and $a$. 
Finally, for any interpreter with respect to $s i$ and natural number $k$, let $\mathbf{F} i k=$ the set of all $k$-level formulas of $s f$ such that

(1) for any variable of $s v$ and assigner in $U i$ with respect to $s a$, if $v$ is not free in $f$, then either $U i$ is empty and ( $f$ satisfies $i$ and $a$ just in case $f$ satisfies $i$ and $a\left(\begin{array}{c}\nu \\ \text { the empty set }\end{array}\right)$ ) or $U i$ is not empty and, for any $m$ in $\mathrm{U} i, f$ satisfies $i$ and $a$ just in case $f$ satisfies $i$ and $a\left(\begin{array}{l}v \\ \{m\}\end{array}\right)$

(2) for any $k$-level term of $s b$, variable of $s v$, and assigner in $U_{i}$ with respect to $s a$, if either $U i$ is empty or the interpretation of $b$ with respect to $i$ and $a$ is not empty, then the result of properly substituting $b$ for $v$ in $f$ in $s$ satisfies $i$ and $a$ just in case $f$ satisfies $i$ and $a\left(\begin{array}{l}v \\ \text { the interpretation of } b \text { with respect to } i \text { and } a\end{array}\right)$

(3.) for any $k$-level terms of $s b$ and $c$ and assigner in $U i$ with respect to $s a$, if the interpretation of $b$ with respect to $i$ and $a=$ the interpretation of $c$ with respect to $i$ and $a$, then the result of properly substituting $b$ for $c$ in $f$ in $s$ satisfies $i$ and $a$ just in case $f$ satisfies $i$ and $a$.

To establish the assertion, it is sufficient to show that, for any interpreter with respect to $s i$,

(1) for any assigner in $\mathrm{U} i$ with respect to $s a$, E $i$ a satisfies condition (d) of part (2) of definition 26

(2) every 0 -level term of $s$ is in $\mathrm{T} i O$

(3) for any natural number $k$,

(a) if every $k$-level term of $s$ is in $\mathrm{T} i k$, then every $k$-level formula of $s$ is in F $i k$

(b) if every $k$-level formula of $s$ is in $\mathbf{F} i k$, then every $k+1-$ level term of $s$ is in $i k+1$.

5. Axiomless deductive systems are usually called natural deduction systems even if they are not at all natural.

6. This is taken from p. 731 of R. McKeon's edition The Basic Works of Aristotle (New York, 1947). The translation is by W. D. Ross.

7. This concept of degree of adequacy is somewhat similar to the concept of degree of confirmation $c^{*}$ discussed by $\mathrm{R}$. Carnap in The Logical Foundations of Probability (Chicago, 1950) and in The Continuum of Inductive Methods (Chicago, 1952).

8. See, for instance, R. Braithwaite's Scientific Explanation (Cambridge, 1953), R. Carnap's 'Foundations of logic and mathematics' (The International Encyclopedia of Unifiea Science, v. 1, 1939), and C. Hempel's 'Fundamentals of concept formation in empirical science' (ibid., v. 2, 1952).

9. An analogue to this concept of concreteness was used by the author as a concept of empirical significance for formulas in 'Some definitions of subjunctive implication, of counterfactual implication, and of related concepts' (Notre Dame Journal of Formal Logic, v. 2, 1961). In connection with this paper, see also the author's 'A note on subjunctive and counterfactual implication' (ibid., v. 3, 1962). 
10. For instance, the three authors of note 8 (in the publications cited in that note).

11. The motivations for and the attributes of denotations and meanings of this kind were more fully discussed by the author in 'A definition of even and some of its applications' (Theoria, v. 28, 1962).

Stockholm, Sweden 